TRANSACTIONS OF THE

AMERICAN MATHEMATICAL SOCIETY

Volume 365, Number 11, November 2013, Pages 5833-5857

S 0002-9947(2013)05792-3

Article electronically published on August 2, 2013

\title{
THE RELATIVE HOCHSCHILD-SERRE SPECTRAL SEQUENCE AND THE BELKALE-KUMAR PRODUCT
}

\author{
SAM EVENS AND WILLIAM GRAHAM
}

\begin{abstract}
We consider the Belkale-Kumar cup product $\odot_{t}$ on $H^{*}(G / P)$ for a generalized flag variety $G / P$ with parameter $t \in \mathbb{C}^{m}$, where $m=$ $\operatorname{dim}\left(H^{2}(G / P)\right)$. For each $t \in \mathbb{C}^{m}$, we define an associated parabolic subgroup $P_{K} \supset P$. We show that the ring $\left(H^{*}(G / P), \odot_{t}\right)$ contains a graded subalgebra $A$ isomorphic to $H^{*}\left(P_{K} / P\right)$ with the usual cup product, where $P_{K}$ is a parabolic subgroup associated to the parameter $t$. Further, we prove that $\left(H^{*}\left(G / P_{K}\right), \odot_{0}\right)$ is the quotient of the ring $\left(H^{*}(G / P), \odot_{t}\right)$ with respect to the ideal generated by elements of positive degree of $A$. We prove the above results by using basic facts about the Hochschild-Serre spectral sequence for relative Lie algebra cohomology, and most of the paper consists of proving these facts using the original approach of Hochschild and Serre.
\end{abstract}

\section{INTRODUCTION}

Let $G$ be a complex semisimple algebraic group with parabolic subgroup $P$ of $G$. Let $m=\operatorname{dim}\left(H^{2}(G / P)\right)$. For each $t \in \mathbb{C}^{m}$, Belkale and Kumar defined a product $\odot_{t}$ which degenerates the usual cup product on $H^{*}(G / P)$, and gave striking applications of this product to the eigenvalue problem and to the problem of finding $G$-invariants in tensor products of representations BK. In EG, we gave a new construction of this product, and showed that the ring $\left(H^{*}(G / P, \mathbb{C}), \odot_{t}\right)$ is isomorphic to a relative Lie algebra cohomology ring $H^{*}\left(\mathfrak{g}_{t}, \mathfrak{l}_{\Delta}\right)$; here $\mathfrak{g}_{t} \supset \mathfrak{l}_{\Delta}$ are certain subalgebras of $\mathfrak{g} \times \mathfrak{g}$.

In this paper, we study the ring $H^{*}\left(G / P, \odot_{t}\right)$. Let $\alpha_{1}, \ldots, \alpha_{n}$ be the simple roots with respect to a Borel subgroup $B \subset P$ and a Cartan subgroup $H \subset B$. Let $L$ be the Levi factor of $P$ containing $H$, let $\mathfrak{l}$ be the Lie algebra of $L$, and number the simple roots so that $I=\left\{\alpha_{m+1}, \ldots, \alpha_{n}\right\}$ are roots of $\mathfrak{l}$, and $\alpha_{1}, \ldots, \alpha_{m}$ are roots of $\mathfrak{u}$, the nilradical of the Lie algebra of $P$. For each $t=\left(t_{1}, \ldots, t_{m}\right) \in \mathbb{C}^{m}$, let $J(t)=\left\{1 \leq q \leq m: t_{q} \neq 0\right\}$, and let $K=J(t) \cup I$. Let $\mathfrak{l}_{K}$ be the Levi subalgebra generated by the Lie algebra $\mathfrak{h}$ of $H$ and the root spaces $\mathfrak{g}_{ \pm \alpha_{i}}$ for $i \in K$, and let $L_{K}$ be the corresponding subgroup. Let $P_{K}=B L_{K}$ be the corresponding standard parabolic.

Theorem 1.1. For parabolic subgroups $P \subset P_{K}$ of $G$, with $P_{K}$ determined by $t \in \mathbb{C}^{m}$ as above,

(1) $H^{*}\left(P_{K} / P\right)$ is isomorphic to a graded subalgebra $A$ of $\left(H^{*}(G / P), \odot_{t}\right)$.

(2) The ring $\left(H^{*}\left(G / P_{K}\right), \odot_{0}\right) \cong\left(H^{*}(G / P), \odot_{t}\right) / I_{+}$, where $I_{+}$is the ideal of $\left(H^{*}(G / P), \odot_{t}\right)$ generated by positive degree elements of $A$.

Received by the editors January 1, 2012.

2010 Mathematics Subject Classification. Primary 17B56, 14M15, 20 G05.

The first author was supported by the National Security Agency. 
This theorem asserts in effect that $\left(H^{*}(G / P), \odot_{t}\right)$ has a classical part isomorphic to the usual cohomology ring, with its associated quotient given by the degenerate Belkale-Kumar product. Using the relative Lie algebra cohomology description of the product, this theorem follows from the Hochschild-Serre spectral sequence in relative Lie algebra cohomology. To make this argument rigorous, one needs to know certain facts about the spectral sequence. In particular, one must show that the spectral sequence degenerates at the $E_{2}$-term, compute the edge morphisms, and determine the product structure on the $E_{2}$-term. The main point of this paper is to carry this out using the original approach of Hochschild and Serre [HS].

While Borel and Wallach give an excellent treatment of representation theoretic aspects of the Hochschild-Serre spectral sequence in [BW], they do not discuss the ring structure, and we are aware of no general reference for the facts needed to prove our theorem. The Hochschild-Serre spectral sequence is of general utility, and one of the goals of this paper is to provide a careful treatment of these facts for the literature. Also, the construction of the relative Hochschild-Serre spectral sequence in $\mathrm{BW}$ uses the identification of the relative Lie algebra cohomology groups with derived functors. In EG the definition of these groups using cochains was crucial in order to be able to apply results of Kostant from [Kos2. By proving what we need using the cochain definition, we avoid the necessity of defining the ring structure in the Borel-Wallach setting, and of proving the compatibility of the two settings.

The approach of Hochschild and Serre generalizes in a mostly straightforward fashion to the relative setting, but there is one new point. In this setting we have a Lie algebra $\mathfrak{g}$, an ideal $I$ and a subalgebra $\mathfrak{k}$ which is reductive in $\mathfrak{g}$. To construct the spectral sequence we need an action of $\mathfrak{g} / I$ on the relative cohomology group $H^{*}(I, I \cap \mathfrak{k} ; M)$ (here $M$ is a $\mathfrak{g}$-module). If $I \cap \mathfrak{k}$ is nonzero, the Lie algebra $\mathfrak{g} / I$ does not act in an obvious way on the space of cochains $C^{*}(I, I \cap \mathfrak{k} ; M)$. Nevertheless, we are able to define the action on the cohomology group by a formula involving cochains; verifying that this does yield the $d_{1}$ differential in the spectral sequence is the main technical complication of the paper. In fact, in the Belkale-Kumar application, $I \cap \mathfrak{k}=0$, so this complication can be avoided, but it seems worthwhile to develop the spectral sequence without this potentially limiting assumption.

The contents of the paper are as follows. In Section 2 we give basic facts about a Lie algebra $\mathfrak{g}$ equipped with $I$ and $\mathfrak{k}$. We also recall some basic definitions related to Lie algebra cohomology, and define the action of $\mathfrak{g} / I$ on $H^{*}(I, I \cap \mathfrak{k} ; M)$. In Section 3 we study the filtration on cochains introduced by Hochschild and Serre and extend their results to the relative setting. In Section 4 we prove some formulas involving differentials which are used in calculating the differentials in the spectral sequence. Section 5 recalls basic definitions and facts about spectral sequences. Section 6] proves the existence of the Hochschild-Serre spectral sequence in the relative setting, identifies the edge maps and proves some results on the product structure. Finally, Section 7 applies these results to the Belkale-Kumar product.

\section{Preliminaries}

We work over a field $\mathbb{F}$ of arbitrary characteristic. In Section 7 we take $\mathbb{F}=\mathbb{C}$. Let $\mathfrak{g}$ be a Lie algebra, $I \subset \mathfrak{g}$ an ideal, and $\mathfrak{k} \subset \mathfrak{g}$ a subalgebra. Write $I_{\mathfrak{k}}=I \cap \mathfrak{k}$. We assume that $\mathfrak{k}$ is reductive in $\mathfrak{g}$. This is equivalent to the statement that if $U \subset V$

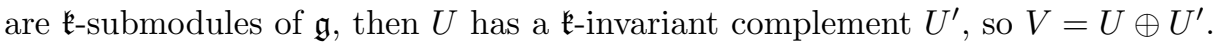


Lemma 2.1. There is a $\mathfrak{k}$-module decomposition of $\mathfrak{g}$ given by

$$
\mathfrak{g}=I_{\mathfrak{k}} \oplus I_{L} \oplus J_{\mathfrak{k}} \oplus J_{L},
$$

such that

$$
I=I_{\mathfrak{k}} \oplus I_{L} \text { and } \mathfrak{k}=I_{\mathfrak{k}} \oplus J_{\mathfrak{k}} .
$$

Moreover, $J:=J_{\mathfrak{k}} \oplus J_{L}$ commutes with $I_{\mathfrak{k}}$.

Proof. Let $I_{L}$ be a $\mathfrak{k}$-module complement of $I_{\mathfrak{k}}$ in $I$, and let $J_{\mathfrak{k}}$ be a $\mathfrak{k}$-module complement of $I_{\mathfrak{k}}$ in $\mathfrak{k}$. Then

$$
I+\mathfrak{k}=I_{\mathfrak{k}} \oplus I_{L} \oplus J_{\mathfrak{k}} .
$$

Let $J_{L}$ be a $\mathfrak{k}$-module complement of $I+\mathfrak{k}$ in $\mathfrak{g}$. The decomposition

$$
\mathfrak{g}=I_{\mathfrak{k}} \oplus I_{L} \oplus J_{\mathfrak{k}} \oplus J_{L}
$$

satisfies (2.2). Moreover, $\left[J, I_{\mathfrak{k}}\right] \subset J \cap I=0$, so $J$ and $I_{\mathfrak{k}}$ commute.

We do not assert that $J$ is a subalgebra of $\mathfrak{g}$, only a $\mathfrak{k}$-submodule. We have a $\mathfrak{k}$-module decomposition $\mathfrak{g}=I \oplus J$. Let $\pi: \mathfrak{g} \rightarrow I$ denote the projection arising from this decomposition. We will sometimes write $x^{*}$ for $\pi(x)$. Let $x \mapsto x^{+}=x-x^{*}$ denote the projection $\mathfrak{g} \rightarrow J$.

Corollary 2.3. The map $\pi: \mathfrak{g} \rightarrow I$ is $\mathfrak{k}$-equivariant, so if $x \in \mathfrak{k}, y \in \mathfrak{g}$, then $[x, \pi(y)]=\pi([x, y])$, or in other words, $\left[x, y^{*}\right]=[x, y]^{*}$. Moreover, $\left.\pi\right|_{I}=i d$ and $\pi(\mathfrak{k})=I_{\mathfrak{k}}$.

The proof is immediate.

2.1. Lie algebra cohomology. In this section we recall some of the basic definitions of Lie algebra cohomology. Let $M$ be a $\mathfrak{g}$-module, and let

$$
C^{n}(\mathfrak{g} ; M)=\operatorname{Hom}\left(\Lambda^{n} \mathfrak{g}, M\right) .
$$

We identify this space with the space of alternating $n$-linear maps from $\mathfrak{g} \times \cdots \times \mathfrak{g}$ to $M$. Given $\underline{x}=\left(x_{1}, \ldots, x_{n}\right) \in \mathfrak{g}^{n}$ and $z \in \mathfrak{g}$, write

$$
f(\underline{x})=f\left(x_{1}, \ldots, x_{n}\right)
$$

and

$$
f(z \cdot \underline{x})=\sum_{i=1}^{n} f\left(x_{1}, \ldots,\left[z, x_{i}\right], \ldots, x_{n}\right) .
$$

The Lie algebra cohomology differential $d: C^{n}(\mathfrak{g} ; M) \rightarrow C^{n+1}(\mathfrak{g} ; M)$ is

$$
\begin{aligned}
d f\left(x_{0}, \ldots, x_{n}\right) & =\sum_{i \leq n}(-1)^{i} x_{i} f\left(x_{0}, \ldots, \hat{x}_{i}, \ldots, x_{n}\right) \\
& +\sum_{i<j \leq n}(-1)^{i+j} f\left(\left[x_{i}, x_{j}\right], x_{0}, \ldots, \hat{x}_{i} \ldots \hat{x}_{j} \ldots, x_{n}\right) .
\end{aligned}
$$

We remark that for any vector space $V$ and any subspace $W \subset V$ we always view $C^{n}(\mathfrak{g} / I ; V)=\operatorname{Hom}_{\mathbb{F}}\left(\Lambda^{n}(\mathfrak{g} / I), V\right)$ as a subspace of $C^{n}(\mathfrak{g} ; V)$, so as arguments to $f \in C^{n}(\mathfrak{g} / I ; V)$, we can allow elements of $\mathfrak{g}$ or of $\mathfrak{g} / I$. We adopt the analogous viewpoint for other spaces.

Define $\theta_{z}: C^{n}(\mathfrak{g} ; M) \rightarrow C^{n}(\mathfrak{g} ; M)$ and $i_{x}: C^{n}(\mathfrak{g} ; M) \rightarrow C^{n-1}(\mathfrak{g} ; M)$ by

$$
\begin{aligned}
\left(\theta_{z} f\right)(\underline{x}) & =z \cdot(f(\underline{x}))-f(z \cdot \underline{x}), \\
\left(i_{z} f\right)\left(x_{2}, \ldots, x_{n}\right) & =f\left(z, x_{2}, \ldots, x_{n}\right) .
\end{aligned}
$$


Then

$$
\theta_{z}=d i_{z}+i_{z} d
$$

We will often write $z \cdot f$ for $\theta_{z} f$. Observe that since $I$ is an ideal of $\mathfrak{g}$, the same formula defines $\theta_{z}: C^{n}(I ; M) \rightarrow C^{n}(I ; M)$.

Lemma 2.7. The differentials on $C(\mathfrak{g} ; M)$ and on $C(I ; M)$ commute with the $\mathfrak{g}$ action.

Proof. The pullback $\gamma^{*}: C(\mathfrak{g} ; M) \rightarrow C(I ; M)$ is surjective and commutes with $d$ and with the $\mathfrak{g}$ action. Therefore it suffices to check that the $\mathfrak{g}$-action on $C(\mathfrak{g} ; M)$ commutes with $d$. The element $z \in \mathfrak{g}$ acts by $\theta_{z}$, and the fact that $\theta_{z}$ commutes with $d$ is an immediate consequence of (2.6).

Let $C^{n}(\mathfrak{g}, \mathfrak{k} ; M)$ denote the subspace of $C^{n}(\mathfrak{g} ; M)$ consisting of those elements annihilated by $i_{x}$ and $\theta_{x}$ for all $x \in \mathfrak{k}$, i.e., the subset of linear maps $f: \Lambda^{n}(\mathfrak{g} / \mathfrak{k}) \rightarrow M$ such that

$$
\theta_{x} \cdot f=0
$$

for all $x \in \mathfrak{k}$. In other words, we can identify

$$
C^{n}(\mathfrak{g}, \mathfrak{k} ; M)=\operatorname{Hom}_{\mathfrak{k}}\left(\Lambda^{n}(\mathfrak{g} / \mathfrak{k}) ; M\right) .
$$

Let

$$
C(\mathfrak{g}, \mathfrak{k} ; M)=\bigoplus_{n} C^{n}(\mathfrak{g}, \mathfrak{k} ; M) .
$$

It is well known that $C(\mathfrak{g}, \mathfrak{k} ; M)$ is a subcomplex of $C(\mathfrak{g} ; M)$.

Let $Z^{q}(\mathfrak{g}, \mathfrak{k} ; M)$ and $B^{q}(\mathfrak{g}, \mathfrak{k} ; M)$ denote the groups of cocycles and coboundaries in $C^{q}(\mathfrak{g}, \mathfrak{k} ; M)$. The $q$-th cohomology group of the complex $C(\mathfrak{g}, \mathfrak{k} ; M)$ is denoted by $H^{q}(\mathfrak{g}, \mathfrak{k} ; M)$. In the absolute case, $Z^{q}(\mathfrak{g} ; M)$ and $B(\mathfrak{g} ; M)$ denote the groups of cocycles and coboundaries in $C^{q}(\mathfrak{g} ; M)$, and $H^{q}(\mathfrak{g} ; M)$ the cohomology. We adopt analogous notation for other Lie algebras.

Pairings on relative Lie algebra cohomology are discussed in Section 6.4

2.2. The $\mathfrak{g} / I$-action on $H^{*}\left(I, I_{\mathfrak{k}} ; M\right)$. In this section we define an action of $\mathfrak{g} / I$ on $H^{q}\left(I, I_{\mathfrak{k}} ; M\right)$ using the complex $C\left(I, I_{\mathfrak{k}} ; M\right)$ (Borel and Wallach define a $\mathfrak{g} / I$-action using injective resolutions).

Lemma 2.8. If $x \in J$, then the operator $\theta_{x}$ on $C^{q}(I ; M)$ preserves the subspace $C^{q}\left(I, I_{\mathfrak{k}} ; M\right)$.

Proof. Let $c \in C^{q}\left(I, I_{\mathfrak{k}} ; M\right)$. We must check that for $y \in I_{\mathfrak{k}}$, we have $\theta_{y} \theta_{x} c=0$ and $i_{y} \theta_{x} c=0$. The first equation holds because $J$ and $I_{\mathfrak{k}}$ commute (Lemma 2.1); thus, $\theta_{y}$ and $\theta_{x}$ commute, so $\theta_{y} \theta_{x} c=\theta_{x} \theta_{y} c=0$, as $\theta_{y} c=0$. To check the second equation, let $y_{1} \in I_{\mathfrak{k}}$, and $y_{2}, \ldots, y_{q} \in I$. Then

$$
\left(i_{y_{1}} \theta_{x} c\right)\left(y_{2}, \ldots, y_{q}\right)=x\left(c\left(y_{1}, \ldots, y_{q}\right)\right)+\sum_{i=1}^{q} c\left(y_{1}, \ldots,\left[y_{i}, x\right], \ldots, y_{q}\right) .
$$

The term $c\left(\left[y_{1}, x\right], y_{2}, \ldots, y_{q}\right)$ is zero because $\left[y_{1}, x\right]=0$. All other terms have $y_{1}$ as an argument of $c$; as $i_{y_{1}} c=0$, these terms give 0 . 
If $x \in J$, then $\theta_{x}$ commutes with the differential $d$ on $C^{q}(I ; M)$ (by Lemma 2.7) and preserves the subspace $C^{q}\left(I, I_{\mathfrak{k}} ; M\right)$ of $C^{q}(I ; M)$. Hence $\theta_{x}$ preserves the subspaces $Z^{q}\left(I, I_{\mathfrak{k}} ; M\right)$ and $B^{q}\left(I, I_{\mathfrak{k}} ; M\right)$, so there is an induced action of $\theta_{x}$ on the cohomology $H^{q}\left(I, I_{\mathfrak{k}} ; M\right)$.

As noted previously, we will often simply write $x \cdot c$ for $\theta_{x} c$. Recall the projection $\mathfrak{g} \rightarrow J, x \mapsto x^{+}$.

Proposition 2.9. There is a Lie algebra action of $\mathfrak{g}$ on $H^{q}\left(I, I_{\mathfrak{k}} ; M\right)$, defined by the formula $x \cdot[c]:=\left[x^{+} \cdot c\right]$ (where $x^{+} \cdot c$ means $\left.\theta_{x^{+}} c\right)$. Since I acts trivially, this formula defines an action of $\mathfrak{g} / I$ on $H^{q}\left(I, I_{\mathfrak{k}} ; M\right)$.

Proof. We must check that the above definition is compatible with the Lie bracket, i.e., that for $x, y \in \mathfrak{g}, c \in Z^{q}\left(I, I_{\mathfrak{k}} ; M\right)$, we have

$$
x \cdot(y \cdot[c])-y \cdot(x \cdot[c])=[x, y] \cdot[c] .
$$

The left hand side is $\left[\left(x^{+} \cdot y^{+}-y^{+} x^{+}\right) c\right]=\left[\left[x^{+}, y^{+}\right] \cdot c\right]$. The right hand side is $\left[[x, y]^{+} \cdot c\right]$. Let $u=\left[x^{+}, y^{+}\right]-[x, y]^{+}$. We must show that $[u \cdot c]=0$, that is, that $u \cdot c \in B^{q}\left(I, I_{\mathfrak{k}} ; M\right)$.

First, observe that $u \in I$. Indeed,

$$
[x, y]=\left[x^{*}+x^{+}, y^{*}+y^{+}\right]=\left[x^{*}, y^{*}\right]+\left[x^{*}, y^{+}\right]+\left[x^{+}, y^{*}\right]+\left[x^{+}, y^{+}\right] .
$$

On the right hand side, all terms but the last are in $I$; hence $[x, y]-\left[x^{+}, y^{+}\right] \in I$. On the other hand, $[x, y]-[x, y]^{+} \in I$. Therefore, $u=\left([x, y]-[x, y]^{+}\right)-([x, y]-$ $\left.\left[x^{+}, y^{+}\right]\right) \in I$.

Next, observe that $u$ commutes with $I_{\mathfrak{k}}$. As by Lemma 2.1, $x^{+}, y^{+}$, and $[x, y]^{+}$ all commute with $I_{\mathfrak{k}}$. We conclude that $u \in Z_{I}\left(I_{\mathfrak{k}}\right)$.

Since $d c=0$, we have

$$
u \cdot c=\left(d i_{u} c-i_{u} d c\right)=d i_{u} c .
$$

Thus, $u \cdot c \in B^{q}(I ; M)$, and to show that $u \cdot c \in B^{q}\left(I, I_{\mathfrak{k}}: M\right)$, we must show that the element $i_{u} c$ of $C^{q-1}(I ; M)$ lies in the subspace $C^{q-1}\left(I, I_{\mathfrak{k}} ; M\right)$. In other words, we must show that for $a \in I_{\mathfrak{k}}$, we have $i_{a} i_{u} c=0$ and $\theta_{a} i_{u} c=0$. The first equation holds because $i_{a} i_{u} c=-i_{u} i_{a} c=0$ as $i_{a} c=0$. To verify the second equation, observe that

$$
\theta_{a} i_{u} c=\left(d i_{a}+i_{a} d\right) i_{u} c=i_{a} d i_{u} c=i_{a} \theta_{u} c,
$$

where in the second equality we have used $i_{a} i_{u} c=0$, and in the third equality we have used $d c=0$. If $y_{2}, \ldots, y_{q} \in I$, then

$$
\begin{aligned}
\left(i_{a} \theta_{u} c\right)\left(y_{2}, \ldots, y_{q}\right)=\left(\theta_{u} c\right)\left(a, y_{2}, \ldots, y_{q}\right)= & u\left(c\left(a, y_{2}, \ldots, y_{q}\right)\right)+c\left([a, u], y_{2}, \ldots, y_{q}\right) \\
& +\sum_{i=2}^{q} c\left(a, y_{2}, \ldots,\left[y_{i}, a\right], \ldots, y_{q}\right) .
\end{aligned}
$$

This is 0 because $i_{a} c=0$ (so we get 0 if any input to $c$ is equal to $a$ ) and $[a, u]=$ 0 . Hence $\theta_{a} i_{u} c=0$. We conclude that $i_{u} c \in C^{q-1}\left(I, I_{\mathfrak{k}} ; M\right)$, so $\theta_{u} c=d i_{u} c \in$ $B^{q}\left(I, I_{\mathfrak{k}} ; M\right)$, completing the proof.

Proposition 2.11. Let $j:\left(I, I_{\mathfrak{k}}\right) \rightarrow(\mathfrak{g}, \mathfrak{k})$ denote the inclusion. The pullback $j^{*}: H^{n}(\mathfrak{g}, \mathfrak{k} ; M) \rightarrow H^{n}\left(I, I_{\mathfrak{k}} ; M\right)$ has image in $H^{n}\left(I, I_{\mathfrak{k}} ; M\right)^{\mathfrak{g} / I}$.

Proof. Let $c \in C^{n}(\mathfrak{g}, \mathfrak{k} ; M)$ be a cocycle. We must show that $j^{*}[c]$ is $\mathfrak{g} / I$-invariant, i.e. if $x \in \mathfrak{g}$, then $x \cdot\left(j^{*}[c]\right)=0$. By definition,

$$
x \cdot\left(j^{*}[c]\right)=\left[\theta_{x} j^{*} c\right] .
$$


So we want to show that $\theta_{x^{+}} j^{*} c$ is the coboundary of an element in $C^{n-1}\left(I, I_{\mathfrak{k}} ; M\right)$. We have

$$
\theta_{x^{+}} j^{*} c=j^{*} \theta_{x^{+}} c,
$$

provided the equation is interpreted correctly: $c \in C^{n}(\mathfrak{g}, \mathfrak{k} ; M) \subset C^{n}(\mathfrak{g} ; M)$, but since we do not know that $C^{n}(\mathfrak{g}, \mathfrak{k} ; M)$ is stable under $\theta_{x^{+}}$, we must view $\theta_{x^{+}} c$ as an element of $C^{n}(\mathfrak{g} ; M)$. We have

$$
j^{*} \theta_{x^{+}} c=j^{*}\left(d i_{x^{+}}+i_{x^{+}} d\right) c=j^{*} d i_{x^{+}} c=d j^{*} i_{x^{+}} c .
$$

We know that $j^{*} i_{x^{+}} c$ is an element of $C^{n-1}(I ; M)$. To complete the proof, we must show that it lies in the subspace $C^{n-1}\left(I, I_{\mathfrak{k}} ; M\right)$. Let $y \in I_{\mathfrak{k}}$. Then $i_{y} j^{*} i_{x^{+}} c=$ $-j^{*} i_{x^{+}} i_{y} c$, and this is zero since $c \in C^{n}(\mathfrak{g}, \mathfrak{k} ; M)$. Also, given $x_{2}, \ldots, x_{n} \in I$, we have

$$
\left(\theta_{y} j^{*} i_{x^{+}} c\right)\left(x_{2}, \ldots, x_{n}\right)=y \cdot\left(c\left(x^{+}, x_{2}, \ldots, x_{n}\right)\right)+\sum_{i \geq 2} c\left(x^{+}, x_{2}, \ldots,\left[x_{i}, y\right], \ldots, x_{n}\right) .
$$

Lemma 2.1 implies that $\left[x^{+}, y\right]=0$, so the right side of (2.13) is unchanged if we add the term $c\left(\left[x^{+}, y\right], x_{2}, \ldots, x_{n}\right)$. But if we add this term, the right hand side of (2.13) is by definition equal to $\left(\theta_{y} c\right)\left(x^{+}, x_{2}, \ldots, x_{n}\right)$. But $\theta_{y} c=0$ since $c \in C^{n}(\mathfrak{g}, \mathfrak{k} ; M)$. We conclude that $j^{*} i_{x^{+}} c$ is in $C^{n-1}(I ; M)$, completing the proof.

\section{The filtration And CONSEQUences}

For simplicity, except for Section 5, we will now write $C^{n}=C^{n}(\mathfrak{g}, \mathfrak{k} ; M)$. This space has a filtration (introduced by Hochschild and Serre in the absolute setting): by definition, $F_{p} C^{n}(\mathfrak{g} ; M)$ consists of the subspace of $C^{n}(\mathfrak{g} ; M)$ consisting of those $f$ which are zero when $n-p+1$ of the inputs are in $I$. In other words, $f\left(x_{1}, \ldots, x_{n}\right)$ can be nonzero only if at most $n-p$ of the $x_{1}, \ldots, x_{n}$ are in $I$. Given a subspace $V$ of $C^{n}(\mathfrak{g} ; M)$, we define $F_{p} V=V \cap F_{p} C^{n}(\mathfrak{g} ; M)$. In particular, this defines $F_{p} C^{n}$. As observed by Hochschild and Serre, the Lie algebra cohomology differential $d$ takes $F_{p} C^{n}(\mathfrak{g} ; M)$ to $F_{p} C^{n+1}(\mathfrak{g} ; M)$. Hence $d$ takes $F_{p} C^{n}$ to $F_{p} C^{n+1}$.

We define $E_{0}^{p q}=F_{p} C^{p+q} / F_{p+1} C^{p+q}$. This is the 0-th page of the HochschildSerre spectral sequence (we recall spectral sequence generalities in Section 5 ). Our immediate goal is to describe $E_{0}^{p q}$.

Since $\mathfrak{k} / I_{\mathfrak{k}}$ acts on $\mathfrak{g} / I$, we can define the vector space

$$
C^{p}\left(\mathfrak{g} / I, \mathfrak{k} / I_{\mathfrak{k}} ; C^{q}\left(I, I_{\mathfrak{k}} ; M\right)\right)=\operatorname{Hom}_{\mathfrak{k} / I_{\mathfrak{k}}}\left(\Lambda^{p}\left(\frac{\mathfrak{g} / I}{\mathfrak{k} / I_{\mathfrak{k}}}\right), C^{q}\left(I, I_{\mathfrak{k}} ; M\right)\right) .
$$

Note that

so

$$
\frac{\mathfrak{g} / I}{\mathfrak{k} / I_{\mathfrak{k}}} \cong \mathfrak{g} /(I+\mathfrak{k})
$$

$$
C^{p}\left(\mathfrak{g} / I, \mathfrak{k} / I_{\mathfrak{k}} ; C^{q}\left(I, I_{\mathfrak{k}} ; M\right)\right)=\operatorname{Hom}_{\mathfrak{k} / I_{\mathfrak{k}}}\left(\Lambda^{p}(\mathfrak{g} /(I+\mathfrak{k})), C^{q}\left(I, I_{\mathfrak{k}} ; M\right)\right) .
$$

Definition 3.2. Let $R_{p}$ denote the map $C^{p+q}(\mathfrak{g} ; M) \rightarrow C^{p}\left(\mathfrak{g} ; C^{q}(I ; M)\right)$ defined by

$$
\left(R_{p} f\right)\left(x_{1}, \ldots, x_{p}\right)\left(x_{p+1}, \ldots, x_{p+q}\right)=f\left(x_{1}, \ldots, x_{p+q}\right),
$$

where $x_{1}, \ldots, x_{p} \in \mathfrak{g}$ and $x_{p+1}, \ldots, x_{p+q} \in I$. Let $S_{p}$ denote the restriction of $R_{p}$ to the subspace $C^{p+q}=C^{p+q}(\mathfrak{g}, \mathfrak{k} ; M)$ of $C^{p+q}(\mathfrak{g} ; M)$. Let $r_{p}$ denote the restriction of $R_{p}$ to $F_{p} C^{p+q}(\mathfrak{g} ; M)$, and $s_{p}$ the restriction of $S_{p}$ to $F_{p} C^{p+q}$. 
Lemma 3.3. $S_{p}$ takes $C^{p+q}$ to $C^{p}\left(\mathfrak{g}, \mathfrak{k} ; C^{q}(I ; M)\right)$.

Proof. We must show that if $f$ is $\mathfrak{k}$-invariant, then so is $S_{p} f$. Let $z \in \mathfrak{k}, x_{1}, \ldots, x_{p} \in$ $\mathfrak{g}$, and $y_{1}, \ldots, y_{q} \in I$. Now, $\mathfrak{k}$-equivariance means that if $z \in \mathfrak{k}$ and $\underline{x} \in \mathfrak{g}^{p}$, then

$$
z \cdot\left[\left(S_{p} f\right)(\underline{x})\right]=\left(S_{p} f\right)(z \cdot \underline{x}) .
$$

To verify this equation, we must evaluate both sides at $\underline{y} \in \mathfrak{g}^{q}$. The left side gives

$$
-f(\underline{x}, z \cdot \underline{y})+z \cdot f(\underline{x}, \underline{y})
$$

while the right side gives

$$
f(z \cdot \underline{x}, \underline{y}) .
$$

The expressions (3.5) and (3.6) are equal because $f$ is $\mathfrak{k}$-equivariant. Hence $S_{p} f$ is $\mathfrak{k}$-equivariant, as desired.

We clarify some notation regarding interior products. Note that if

$$
f \in C^{p}\left(\mathfrak{g}, C^{q}(\mathfrak{g} ; M)\right)
$$

and $y \in \mathfrak{g}$, then $i_{y} f \in C^{p-1}\left(\mathfrak{g}, C^{q}(\mathfrak{g} ; M)\right)$. Given a $p$-tuple $\underline{x}=\left(x_{1}, \ldots, x_{j}\right)$, let $i_{x}: C^{p}\left(\mathfrak{g} ; C^{q}(\mathfrak{g} ; M)\right) \rightarrow C^{q}(\mathfrak{g} ; M)$ denote the evaluation map. In this case, if $f \in C^{p}\left(\mathfrak{g} ; C^{q}(\mathfrak{g} ; M)\right)$, then $i_{y} i_{x} f \in C^{q-1}(\mathfrak{g} ; M)$, and $\theta_{y} i_{x} f \in C^{q-1}(\mathfrak{g} ; M)$. We will use the same notation for maps restricted to subspaces of $C^{p+q}(\mathfrak{g} ; M)$ and $C^{p}\left(\mathfrak{g} ; C^{q}(\mathfrak{g} ; M)\right)$.

The following lemma generalizes to the relative situation a result from [HS]; the arguments are adapted from there. The lemma implies that $s_{p}$ induces an isomorphism

$$
E_{0}^{p q}=F_{p} C^{p+q} / F_{p+1} C^{p+q} \cong C^{p}\left(\mathfrak{g} / I, \mathfrak{k} / I_{\mathfrak{k}} ; C^{q}\left(I, I_{\mathfrak{k}} ; M\right)\right) .
$$

This is our desired description of $E_{0}^{p q}$.

Lemma 3.7. (1) The kernel of $s_{p}$ is $F_{p+1} C^{p+q}$.

(2) $s_{p}$ maps $F_{p} C^{p+q}$ to $C^{p}\left(\mathfrak{g} / I, \mathfrak{k} / I_{\mathfrak{k}} ; C^{q}\left(I, I_{\mathfrak{k}} ; M\right)\right)$.

(3) The image of $s_{p}$ is all of $C^{p}\left(\mathfrak{g} / I, \mathfrak{k} / I_{\mathfrak{k}} ; C^{q}\left(I, I_{\mathfrak{k}} ; M\right)\right)$.

Proof. (1) The statement about the kernel of $s_{p}$ is immediate from the definition of the filtration.

(2) Suppose $f \in F_{p} C^{p+q}$. We want to show that $s_{p}(f) \in C^{p}\left(\mathfrak{g} / I, \mathfrak{k} / I_{\mathfrak{k}} ; C^{q}\left(I, I_{\mathfrak{k}} ; M\right)\right)$. Since $s_{p}$ is the restriction of $S_{p}$ and of $r_{p}$, we know that $s_{p} f$ lies in the intersection of the images of $S_{p}$ and of $r_{p}$, i.e., in

$$
C^{p}\left(\mathfrak{g}, \mathfrak{k} ; C^{q}(I ; M)\right) \cap C^{p}\left(\mathfrak{g} / I ; C^{q}(I ; M)\right) .
$$

To prove (2), it will be enough to show that for $\underline{x} \in \mathfrak{g}^{p}$, we have $i_{\underline{x}} f \in C^{q}\left(I, I_{\mathfrak{k}} ; M\right)$, for then the notions of $\mathfrak{k}$-equivariance and $\mathfrak{k} / I_{\mathfrak{k}}$-equivariance will coincide.

To show that $i_{\underline{x}} f \in C^{q}\left(I, I_{\mathfrak{k}} ; M\right)$, we must verify that for $z \in I_{\mathfrak{k}}$, we have

$$
i_{z} i_{\underline{x}} s_{p} f=0
$$

and

$$
\theta_{z} i_{\underline{x}} s_{p} f=0 \text {. }
$$

The first equation holds because if an element of $\mathfrak{k}$ is an argument of $f$, the result is 0 . For the second equation, since $f \in C^{p+q}(\mathfrak{g}, \mathfrak{k} ; M)$, and $z \in I_{\mathfrak{k}} \subset \mathfrak{k}$, we have $\theta_{z} f=0$. By definition of $\theta_{z}$, this means that for $\underline{x} \in \mathfrak{g}^{p}$ and $\underline{y} \in I^{q}$, we have

$$
z \cdot(f(\underline{x}, \underline{y}))=f(z \cdot \underline{x}, \underline{y})+f(\underline{x}, z \cdot \underline{y}) .
$$


The first term on the right is 0 because it is a sum of terms, each of which has at least $q+1$ inputs from $I$. Thus, we obtain

$$
\left[\theta_{z} i_{\underline{x}} s_{p} f\right](\underline{y})=z \cdot(f(\underline{x}, \underline{y}))-f(\underline{x}, z \cdot \underline{y})=0 .
$$

As $y$ is arbitrary, we see that $\theta_{z} i_{\underline{x}} s_{p} f=0$, as desired.

$(\overline{3})$ We now prove that $s_{p}$ is surjective. We must show that if

$$
g \in C^{p}\left(\mathfrak{g} / I, \mathfrak{k} / I_{\mathfrak{k}} ; C^{q}\left(I, I_{\mathfrak{k}} ; M\right)\right),
$$

then there exists $f \in F_{p} C^{p+q}$ such that $s_{p} f=g$.

As in [HS], define $f \in C^{p+q}(\mathfrak{g} ; M)$ by

$$
f\left(x_{1}, \ldots, x_{p+q}\right)=\sum_{\sigma}(-1)^{\sigma} g\left(x_{\sigma(1)}, \ldots, x_{\sigma(p)}\right)\left(x_{\sigma(p+1)}^{*}, \ldots, x_{\sigma(p+q)}^{*}\right) .
$$

Here, the map $x \mapsto x^{*}$ is as in Corollary 2.3. The sum is over all permutations $\sigma \in \Sigma_{p+q}$ such that $\sigma(1)<\sigma(2)<\cdots<\sigma(p)$ and $\sigma(p+1)<\sigma(p+2)<\cdots<$ $\sigma(p+q)$. (We have asserted that $f \in C^{p+q}(\mathfrak{g} ; M)$. This amounts to checking that $f$ is alternating; see Remark 3.11 below.) We must verify that $f \in C^{p+q}(\mathfrak{g}, \mathfrak{k} ; M)$, i.e., that if $x \in \mathfrak{k}$, then $i_{x} f=0$ and $\theta_{x} f=0$. To show the first equation, it suffices to show that if $x_{1} \in \mathfrak{k}$, then $f\left(x_{1}, \ldots, x_{p+q}\right)=0$. This holds because either $\sigma(1)=1$, in which case we get 0 as $i_{x_{1}} g=0$, or $\sigma(p+1)=1$, in which case $x_{1}^{*} \in I_{\mathfrak{k}}$ by Corollary 2.3. so we get 0 because $i_{\underline{x}} g \in C^{q}\left(I, I_{\mathfrak{k}} ; M\right)$.

Now we show that $\theta_{x} f=0$. This says that for $z \in \mathfrak{k}$ and $x_{1}, \ldots, x_{p+q} \in \mathfrak{g}$, we have

$$
\sum_{i=1}^{p+q} f\left(x_{1} \ldots,\left[z, x_{i}\right], \ldots, x_{p+q}\right)=z \cdot\left(f\left(x_{1}, \ldots, x_{p+q}\right)\right) .
$$

By assumption, $g$ is $\mathfrak{k} / I_{\mathfrak{k}}$-equivariant (i.e., $\mathfrak{k}$-invariant, and $I_{\mathfrak{k}}$ acts trivially). Thus, for $z \in \mathfrak{k}$ and $x_{1}, \ldots, x_{p} \in \mathfrak{g}$, we have

$$
\sum_{i=1}^{p} g\left(x_{1}, \ldots,\left[z, x_{i}\right], \ldots, x_{p}\right)=z \cdot\left(g\left(x_{1}, \ldots, x_{p}\right)\right) .
$$

Given $\left(x_{p+1}, \ldots, x_{p+q}\right) \in \mathfrak{g}^{q}$ we have $\left(x_{p+1}^{*}, \ldots, x_{p+q}^{*}\right) \in I^{q}$, to which we can apply both sides of the preceding equation. Upon rearranging, by definition of the $\mathfrak{k}$-action on $C^{q}\left(I, I_{\mathfrak{k}} ; M\right)$, we obtain

$$
\begin{aligned}
& \sum_{i=1}^{p} g\left(x_{1}, \ldots,\left[z, x_{i}\right], \ldots, x_{p}\right)\left(x_{p+1}^{*}, \ldots, x_{p+q}^{*}\right) \\
& \quad+\sum_{j=p+1}^{p+q} g\left(x_{1}, \ldots, x_{p}\right)\left(x_{p+1}^{*} \ldots,\left[z, x_{j}^{*}\right], \ldots, x_{p+q}^{*}\right) \\
& \quad=z \cdot\left(g\left(x_{1}, \ldots, x_{p}\right)\left(x_{p+1}^{*}, \ldots, x_{p+q}^{*}\right)\right) .
\end{aligned}
$$


Now, $\left[z, x_{j}^{*}\right]=\left[z, x_{j}\right]^{*}$ by Corollary 2.3 . Hence

$$
\begin{array}{r}
\sum_{\sigma}(-1)^{\sigma}\left(\sum_{i=1}^{p} g\left(x_{\sigma(1)}, \ldots,\left[z, x_{\sigma(i)}\right], \ldots, x_{\sigma(p)}\right)\left(x_{\sigma(p+1)}^{*}, \ldots, x_{\sigma(p+q)}^{*}\right)\right. \\
\left.+\sum_{j=p+1}^{p+q} g\left(x_{\sigma(1)}, \ldots, x_{\sigma(p)}\right)\left(x_{\sigma(p+1)}^{*} \ldots,\left[z, x_{\sigma(j)}\right]^{*}, \ldots, x_{\sigma(p+q)}^{*}\right)\right) \\
=\sum_{\sigma}(-1)^{\sigma} z \cdot\left(g\left(x_{\sigma(1)}, \ldots, x_{\sigma(p)}\right)\left(x_{\sigma(p+1)}^{*}, \ldots, x_{\sigma(p+q)}^{*}\right)\right) .
\end{array}
$$

This is exactly the equation we obtain by taking the equation (3.9) of $\mathfrak{k}$-equivariance of $f$ and expressing $f$ in terms of $g$. This proves (3.9), so $f$ is k-equivariant. We conclude that $f \in C^{p+q}(\mathfrak{g}, \mathfrak{k} ; M)$, as desired.

Next, observe that $f \in F_{p} C^{p+q}(\mathfrak{g}, \mathfrak{k} ; M)$. The reason is that if more than $q$ of the arguments of $f$ are in $I$, then for any permutation $\sigma$, at least one of the $x_{\sigma(1)}, \ldots, x_{\sigma(p)}$ must be in $I$, in which case $g\left(x_{\sigma(1)}, \ldots, x_{\sigma(p)}\right)\left(x_{\sigma(p+1)}^{*}, \ldots, x_{\sigma(p+q)}^{*}\right)$ $=0$.

Finally, we must verify that $s_{p} f=g$. For this, suppose that $x_{1}, \ldots, x_{p}$ are in $\mathfrak{g}$ and $x_{p+1}, \ldots, x_{p+q}$ are in $I$. Then in the definition of $f$, the only permutation that contributes is the identity permutation, and we see that

$$
f\left(x_{1}, \ldots, x_{p+q}\right)=g\left(x_{1}, \ldots, x_{p}\right)\left(x_{p+1}, \ldots, x_{p+q}\right),
$$

so $s_{p} f=g$, as desired.

Remark 3.11. To show that $f$ is alternating, it suffices to show that if $x_{i}=x_{i+1}$ for some $i$, then $f\left(x_{1}, \ldots, x_{p+q}\right)=0$. Suppose $\sigma \in \Sigma_{p+q}$ is a permutation contributing to the sum (3.8), and suppose that $\sigma(a)=i, \sigma(b)=i+1$. If $a$ and $b$ are both $\leq p$ or both $\geq p+1$, then $g\left(x_{\sigma(1)}, \ldots, x_{\sigma(p)}\right)\left(x_{\sigma(p+1)}^{*}, \ldots, x_{\sigma(p+q)}^{*}\right)=0$ by the alternating properties of $g$. Otherwise, the contribution to (3.8) from $\sigma$ is the negative of the contribution from $\tau \sigma$, where $\tau$ is the transposition $(i i+1)$. We conclude that $f\left(x_{1}, \ldots, x_{p+q}\right)=0$, as desired.

Definition 3.12. For later use, we will denote by $g \mapsto \widetilde{g}$ the map

$$
C^{p}\left(\mathfrak{g} / I, \mathfrak{k} / I_{\mathfrak{k}} ; C^{q}\left(I, I_{\mathfrak{k}} ; M\right)\right) \rightarrow F_{p} C^{p+q}
$$

constructed in the preceding proof; that is, $\widetilde{g}$ is the element $f$ defined in (3.8). The preceding proof shows that $s_{p} \widetilde{g}=S_{p}(\widetilde{g})=g$.

\section{Some FORMUlas INVOLVING DIFFERENTIALS}

Here we give some facts involving various Lie algebra cohomology differentials, which will be used in Section [6 when we calculate the differentials in the spectral sequence.

We let $C^{*, *}=\bigoplus_{p, q \geq 0} C^{p, q}$, where $C^{p, q}=C^{p}\left(\mathfrak{g} ; C^{q}(I ; M)\right)$. Recall from Definition 3.2 the map $R_{p}: C^{p+q}(\mathfrak{g} ; M) \rightarrow C^{p, q}$. There are two differentials on $C^{*, *}$. First, there is the vertical differential $d_{v}: C^{p, q} \rightarrow C^{p, q+1}$. This is defined as $d_{v} f=d \circ f$ for $f \in C^{p}\left(\mathfrak{g} ; C^{q}(I ; M)\right)$, where the $d$ on the right hand side of the equation is the differential $C^{q}(I ; M) \rightarrow C^{q+1}(I ; M)$. Second, there is the horizontal differential $d_{h}: C^{p, q} \rightarrow C^{p+1, q}$ (this is the Lie algebra cohomology differential $d: C^{p}\left(\mathfrak{g} ; C^{q}(I ; M)\right) \rightarrow C^{p+1}\left(\mathfrak{g} ; C^{q}(I ; M)\right)$, with $C^{q}(I ; M)$ playing the role of the module; note that $x \in \mathfrak{g}$ acts on $C^{q}(I ; M)$ using the action $\theta_{x}$ defined above). 
Lemma 4.1. The differentials $d_{h}$ and $d_{v}$ (on $C(\mathfrak{g}, C(I ; M))$ ) commute.

Proof. Any $\mathfrak{g}$-module map $V \rightarrow W$ induces a map of complexes $C(\mathfrak{g} ; V) \rightarrow C(\mathfrak{g} ; W)$ which is compatible with the Lie algebra cohomology differential. By Lemma 2.7. the differential $C^{q}(I ; M) \rightarrow C^{q+1}(I ; M)$ is a $\mathfrak{g}$-module map. The induced map $C\left(\mathfrak{g} ; C^{q}(I ; M)\right) \rightarrow C\left(\mathfrak{g} ; C^{q+1}(I ; M)\right)$ is what we have denoted $d_{v}$, and the compatibility with the Lie algebra cohomology differential (with $\mathfrak{g}$ playing the role of Lie algebra) amounts to the commutativity of $d_{h}$ and $d_{v}$.

Lemma 4.2. The space $C(\mathfrak{g}, \mathfrak{k} ; C(I, M))$ (viewed as a subspace of $C(\mathfrak{g}, C(I, M))$ ) is stable under $d_{h}$ and $d_{v}$. Moreover, the spaces

$$
C\left(\mathfrak{g} / I, \mathfrak{k} / I_{\mathfrak{k}} ; C\left(I, I_{\mathfrak{k}} ; M\right)\right) \subset C\left(\mathfrak{g}, \mathfrak{k} ; C\left(I, I_{\mathfrak{k}} ; M\right)\right)
$$

of $C(\mathfrak{g}, \mathfrak{k} ; C(I, M))$ are stable under $d_{v}$.

Proof. The space $C(\mathfrak{g}, \mathfrak{k} ; C(I, M))$ is stable under $d_{h}$ because it is a relative Lie algebra cohomology complex (with $C(I ; M)$ playing the role of module). To verify stability under $d_{v}$, let $f \in C^{p}\left(\mathfrak{g}, \mathfrak{k} ; C^{q}(I ; M)\right)$, and $z \in \mathfrak{k}$. We must show two things. First, for $z$ in $\mathfrak{k}, d_{v} f \in C^{p}\left(\mathfrak{g} ; C^{i+1}(I ; M)\right)$ is annihilated by $i_{z}$. This follows because $d_{v} f=d \circ f$, and $f$ vanishes when any input is in $\mathfrak{k}$. Second, $d_{v} f: \mathfrak{g}^{p} \rightarrow C^{q}(I ; M)$ is $\mathfrak{k}$-equivariant. Indeed, suppose $z \in \mathfrak{k}$ and $\underline{x} \in \mathfrak{g}^{p}$. Writing the action of $z$ on $C(I ; M)$ as $\theta_{z}$, we want to show that

$$
\left(d_{v} f\right)(z \cdot \underline{x})=\theta_{z}\left(\left(d_{v} f\right)(\underline{x})\right) .
$$

By definition, $\left(d_{v} f\right)(z \cdot \underline{x})=d(f(z \cdot \underline{x}))$. Since $f$ is $\mathfrak{k}$-equivariant, this equals $d\left(\theta_{z}(f(\underline{x}))\right)$. By Lemma 2.7, $d$ commutes with $\theta_{z}$. Hence $C(\mathfrak{g}, \mathfrak{k} ; C(I ; M))$ is stable under $d_{v}$. The remaining assertions follow easily.

The following lemma is essentially given in [HS], so we omit the proof.

Lemma 4.3. Let $f \in C^{p+q}(\mathfrak{g} ; M)$. Then

$$
R_{p+1} d f=d_{h}\left(R_{p} f\right)+(-1)^{p+1} d_{v}\left(R_{p+1} f\right) .
$$

The analogous formula holds with $R$ replaced by $S$.

4.1. Differentials related to relative cohomology. To streamline the exposition we introduce some notation.

Definition 4.5. Write

$$
C^{p}\left(C^{q}\right)=C^{p}\left(\mathfrak{g} / I, \mathfrak{k} / I_{\mathfrak{k}} ; C^{q}\left(I, I_{\mathfrak{k}} ; M\right)\right) .
$$

We adopt the analogous notation when $C^{q}\left(I, I_{\mathfrak{k}} ; M\right)$ is replaced by $Z^{q}\left(I, I_{\mathfrak{k}} ; M\right)$, $B^{q}\left(I, I_{\mathfrak{k}} ; M\right)$ or $H^{q}\left(I, I_{\mathfrak{k}} ; M\right)$ (cocycles, coboundaries, and cohomology, respectively). When we write $C^{p}$ by itself, we will mean $C^{p}(\mathfrak{g}, \mathfrak{k} ; M)$. Also, since $\mathfrak{g} / I$ acts on $H^{q}\left(I, I_{\mathfrak{k}} ; M\right)$, we have groups of coboundaries and cocycles:

$$
B^{p}\left(H^{q}\right):=B^{p}\left(\mathfrak{g} / I, \mathfrak{k} / I_{\mathfrak{k}} ; H^{q}\left(I, I_{\mathfrak{k}} ; M\right)\right) \subset Z^{p}\left(H^{q}\right):=Z^{p}\left(\mathfrak{g} / I, \mathfrak{k} / I_{\mathfrak{k}} ; H^{q}\left(I, I_{\mathfrak{k}} ; M\right)\right)
$$

and the corresponding cohomology group

$$
H^{p}\left(H^{q}\right):=H^{p}\left(\mathfrak{g} / I, \mathfrak{k} / I_{\mathfrak{k}} ; H^{q}\left(I, I_{\mathfrak{k}} ; M\right)\right) .
$$


We need a little more notation. Given a $(p+1)$-tuple $\underline{y}=\left(y_{0}, \ldots, y_{p}\right)$, and $0 \leq i \leq p$, let $\underline{y}(i)$ denote the $p$-tuple $\left(y_{0}, \ldots, \hat{y}_{i}, \ldots, y_{p}\right)$. Given $z \in C^{p}\left(Z^{q}\right)$, we can view $z(y(j))$ as an element of $Z^{q}\left(I, I_{\mathfrak{k}} ; M\right) \subset Z^{q}(I ; M)$. The space $Z^{q}(I ; M)$ has a $\mathfrak{g}$-module structure. To be explicit, given $u \in \mathfrak{g}$ and $y_{p+1}, \ldots, y_{p+q} \in I$, we have

$$
\begin{aligned}
{[u \cdot z(\underline{y}(j))]\left(y_{p+1}, \ldots, y_{p+q}\right) } & =u \cdot\left[z(\underline{y}(j))\left(y_{p+1}, \ldots, y_{p+q}\right)\right] \\
& +\sum_{r=p+1}^{p+q} z(\underline{y}(j))\left(y_{p+1}, \ldots,\left[y_{r}, u\right], \ldots, \ldots, y_{p+q}\right) .
\end{aligned}
$$

Lemma 4.6. There is a map

$$
d_{+}: C^{p}\left(C^{q}\right) \rightarrow C^{p+1}\left(C^{q}\right)
$$

defined by the formula

$$
\begin{aligned}
& \left(d_{+} z\right)\left(y_{0}, \ldots, y_{p}\right)=\sum_{i=0}^{p}(-1)^{i} y_{i}^{+} \cdot z(\underline{y}(i)) \\
& \quad+\sum_{0 \leq i<j \leq p}(-1)^{i+j} z\left(\left[y_{i}, y_{j}\right], y_{0}, \ldots, \hat{y}_{i}, \ldots, \hat{y}_{j}, \ldots, y_{p}\right) .
\end{aligned}
$$

Proof. It is evident that $d_{+} z \in C^{p+1}\left(\mathfrak{g} / I ; C^{q}\left(I, I_{\mathfrak{k}} ; M\right)\right)$. We must verify that $d_{+} z$ is $\mathfrak{k} / I_{\mathfrak{k}}$-equivariant or, equivalently, is $\mathfrak{k}$-equivariant. We have

$$
C^{p}\left(\mathfrak{g} / I, \mathfrak{k} / I_{\mathfrak{k}} ; C^{q}\left(I, I_{\mathfrak{k}} ; M\right)\right) \subset C^{p}\left(\mathfrak{g}, \mathfrak{k} ; C^{q}(I ; M)\right) \stackrel{d_{\mathfrak{h}}}{\rightarrow} C^{p+1}\left(\mathfrak{g}, \mathfrak{k} ; C^{q}(I ; M)\right) .
$$

Define $e: C^{p}\left(\mathfrak{g} / I, \mathfrak{k} / I_{\mathfrak{k}} ; C^{q}\left(I, I_{\mathfrak{k}} ; M\right)\right) \rightarrow C^{p+1}\left(\mathfrak{g} / I ; C^{q}(I ; M)\right)$ by the formula

$$
(e z)\left(y_{0}, \ldots, y_{p}\right)=\sum_{i=0}^{p}(-1)^{i} y_{i}^{*} \cdot(z(\underline{y}(i))) .
$$

Since $d_{h}$ is a relative Lie algebra cohomology differential, $d_{h} z$ is $\mathfrak{k}$-equivariant. Since $d_{+}=d_{h}-e$, it suffices to check that $e z$ is $\mathfrak{k}$-equivariant as well, i.e., that if $u \in \mathfrak{k}$ and $\underline{y}=\left(y_{0}, \ldots, y_{p}\right)$, then

$$
u((e z)(\underline{y}))=(e z)(u \cdot \underline{y}) .
$$

The left hand side of this equation is

$$
\sum_{i=0}^{p}(-1)^{i} u y_{i}^{*} z(\underline{y}(i)) .
$$

The right hand side is

$$
\sum_{i=0}^{p}(e z)\left(y_{0}, \ldots,\left[u, y_{i}\right], \ldots, y_{p}\right) .
$$

This can be rewritten as

$$
\sum_{i=0}^{p}(-1)^{i}\left[u, y_{i}\right]^{*} \cdot z(\underline{y}(i))+\sum_{i=0}^{p} \sum_{j \neq i}(-1)^{j} y_{j}^{*} z\left(y_{0}, \ldots, \hat{y}_{j}, \ldots,\left[u, y_{i}\right], \ldots, y_{p}\right) .
$$

By Corollary $2.3\left[u, y_{i}\right]^{*}=\left[u, y_{i}^{*}\right]$, and this element acts on $z(\underline{y}(i))$ as $u y_{i}^{*}-y_{i}^{*} u$. Also, the double sum in (4.11) can be rewritten as

$$
\sum_{j=0}^{p}(-1)^{j} y_{j}^{*} z(u \cdot \underline{y}(j)) \text {. }
$$


Therefore, changing $j$ to $i$ in this sum, we can rewrite (4.11) as

$$
\sum_{i=0}^{p}(-1)^{i}\left(u y_{i}^{*}-y_{i}^{*} u\right) \cdot z(\underline{y}(i))+\sum_{i=0}^{p}(-1)^{i} y_{i}^{*} z(u \cdot \underline{y}(i)) .
$$

We want to show that this is equal to (4.9), i.e., that

$$
0=-\sum_{i=0}^{p}(-1)^{i} y_{i}^{*} u \cdot z(\underline{y}(i))+\sum_{i=0}^{p}(-1)^{i} y_{i}^{*} z(u \cdot \underline{y}(i)) .
$$

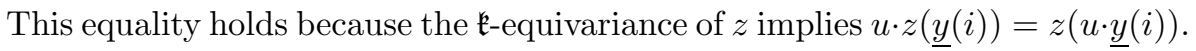

Although $d_{h}$ commutes with $d_{v}$, we do not assert that $d_{+}$commutes with $d_{v}$.

Notice that the formula for $d_{+}$would be a Lie algebra cohomology differential if $\mathfrak{g} / I$ acted on $C^{q}\left(I, I_{\mathfrak{k}} ; M\right)$, with the action of $x$ taking $c$ to $x^{+} \cdot c$. However, this formula does not define a Lie algebra action (i.e., it is not compatible with the Lie bracket). Thus, we cannot assert that $d_{+} \circ d_{+}$is zero on cochains. However, by Proposition 2.9. $\mathfrak{g} / I$ does act on $H^{q}\left(I, I_{\mathfrak{k}} ; M\right)$ by the analogous formula, i.e., by $x \cdot[c]=\left[x^{+} \cdot c\right]$. Write $[z]$ for the image of $z$ under the map

$$
C^{p}\left(\mathfrak{g} / I, \mathfrak{k} / I_{\mathfrak{k}} ; Z^{q}\left(I, I_{\mathfrak{k}} ; M\right)\right) \rightarrow C^{p}\left(\mathfrak{g} / I, \mathfrak{k} / I_{\mathfrak{k}} ; H^{q}\left(I, I_{\mathfrak{k}} ; M\right)\right) .
$$

The Lie algebra cohomology differential

$$
C^{p}\left(\mathfrak{g} / I, \mathfrak{k} / I_{\mathfrak{k}} ; H^{q}\left(I, I_{\mathfrak{k}} ; M\right)\right) \rightarrow C^{p+1}\left(\mathfrak{g} / I, \mathfrak{k} / I_{\mathfrak{k}} ; H^{q}\left(I, I_{\mathfrak{k}} ; M\right)\right)
$$

is given by $[z] \mapsto\left[d_{+} z\right]$. By abuse of notation we will simply write $d_{+}[z]=\left[d_{+} z\right]$.

Lemma 4.12. Let $f \in Z^{q}(I ; M)$, and let $x_{0}, \ldots, x_{q} \in I$. Then

$$
\begin{aligned}
\left(x_{0} \cdot f\right)\left(x_{1}, \ldots, x_{q}\right) & =\sum_{r=1}^{q}(-1)^{r+1} x_{r}\left(f\left(x_{0}, \ldots, \hat{x}_{r}, \ldots, x_{q}\right)\right) \\
& +\sum_{1 \leq r<s}(-1)^{r+s} f\left(x_{0},\left[x_{r}, x_{s}\right], x_{1}, \ldots, \hat{x}_{r}, \ldots, \hat{x}_{s}, \ldots\right) .
\end{aligned}
$$

Proof. This follows by writing out the equation $d f\left(x_{0}, \ldots, x_{q}\right)=0$ and rearranging, using the definition of $x_{0} \cdot f$.

The following proposition and corollary relate $z=S_{p} \widetilde{z}$ and $S_{p+1} \widetilde{z}$.

Proposition 4.13. Let $z \in C^{p}\left(\mathfrak{g} / I, \mathfrak{k} / I_{\mathfrak{k}} ; Z^{q}\left(I, I_{\mathfrak{k}} ; M\right)\right)$, and let $\widetilde{z} \in C^{p+q}$ be defined as in Definition $\lcm{3.12}$ (so $\left.S_{p} \widetilde{z}=z\right)$. Let $\left(y_{0}, \ldots, y_{p}\right) \in \mathfrak{g}^{p+1}$. Then

$$
\left(d_{v} S_{p+1} \widetilde{z}\right)\left(y_{0}, \ldots, y_{p}\right)=(-1)^{p} \sum_{i=0}^{p}(-1)^{i} y_{i}^{*} \cdot z(\underline{y}(i)) .
$$

Proof. First, observe that given $\underline{a}=\left(a_{0}, \ldots, a_{p}\right) \in \mathfrak{g}^{p}$, and $\left(b_{1}, \ldots, b_{q-1}\right) \in I^{q-1}$, we have

$$
\left(S_{p+1} \widetilde{z}\right)\left(a_{0}, \ldots, a_{p}\right)\left(b_{1}, \ldots, b_{q-1}\right)=(-1)^{p} \sum_{i=0}^{p}(-1)^{i} z(\underline{a}(i))\left(a_{i}^{*}, b_{1}, \ldots, b_{q-1}\right) .
$$

This follows from the definition of $\widetilde{z}$, along with the fact that if one of $u_{1}, \ldots, u_{p}$ is in $I$, then $z\left(u_{1}, \ldots, u_{p}\right)\left(v_{1}, \ldots, v_{q}\right)=0$. 
Now let $\left(y_{0}, \ldots, y_{p}\right) \in \mathfrak{g}^{p+1}$ and $\left(y_{p+1}, \ldots, y_{p+q}\right) \in I^{q}$. To simplify the notation, for each $i \leq p$ let $F_{i}=z(\underline{y}(i)) \in Z^{q}(I)$. By definition,

$$
\left(d_{v} S_{p+1} \widetilde{z}\right)\left(y_{0}, \ldots, y_{p}\right)\left(y_{p+1}, \ldots, y_{p+q}\right)
$$

is equal to

$$
\begin{array}{r}
\sum_{p+1 \leq r}(-1)^{r+p+1} y_{r}\left(S_{p+1} \widetilde{z}\right)\left(y_{0}, \ldots, y_{p}\right)\left(y_{p+1}, \ldots, \hat{y}_{r}, \ldots, y_{p+q}\right) \\
+\sum_{p+1 \leq r<s}(-1)^{r+s}\left(S_{p+1} \widetilde{z}\right)\left(y_{0}, \ldots, y_{p}\right)\left(\left[y_{r}, y_{s}\right], y_{p+1}, \ldots, \hat{y}_{r}, \ldots, \hat{y}_{s}, \ldots, y_{p+q}\right) .
\end{array}
$$

Equation (4.14) implies that this is equal to

$$
\begin{gathered}
\sum_{p+1 \leq r} \sum_{i \leq p}(-1)^{r+i+1} y_{r} F_{i}\left(y_{i}^{*}, y_{p+1}, \ldots, \hat{y}_{r}, \ldots\right) \\
+\sum_{p+1 \leq r<s} \sum_{i \leq p}(-1)^{r+s+i+p} F_{i}\left(y_{i}^{*},\left[y_{r}, y_{s}\right], y_{p+1}, \ldots, \hat{y}_{r}, \ldots, \hat{y}_{s}, \ldots, y_{p+q}\right) .
\end{gathered}
$$

We can rearrange this expression to obtain

$$
\begin{aligned}
(-1)^{p} \sum_{i \leq p}(-1)^{i}\left(\sum_{p+1 \leq r}(-1)^{r+1+p} y_{r}\left(F_{i}\left(y_{i}^{*}, y_{p+1}, \ldots, \hat{y}_{r}, \ldots, y_{p+q}\right)\right)\right. & \\
& \left.+\sum_{p+1 \leq r<s}(-1)^{r+s} F_{i}\left(y_{i}^{*},\left[y_{r}, y_{s}\right], \ldots, \hat{y}_{r}, \ldots, \hat{y}_{s}, \ldots, y_{p+q}\right)\right) .
\end{aligned}
$$

It follows from Lemma 4.12, with $F_{i}$ playing the role of $f$ and $y_{i}^{*}, y_{p+1}, \ldots, y_{p+q}$ playing the role of $x_{0}, \ldots, x_{q}$, that the above expression is equal to

$$
(-1)^{p} \sum_{i=0}^{p}(-1)^{i}\left(y_{i}^{*} F_{i}\right)\left(y_{p+1}, \ldots, y_{p+q}\right),
$$

proving the proposition.

Corollary 4.15. Let $z$ and $\widetilde{z}$ be as in Proposition 4.13, Then

$$
s_{p+1} d \widetilde{z}=d_{+} z .
$$

Proof. By definition, $s_{p+1} d \widetilde{z}=S_{p+1} d \widetilde{z}$. By construction, $S_{p} \widetilde{z}=z$. Thus, by Lemma 4.3 .

$$
S_{p+1} d \widetilde{z}=d_{h} z+(-1)^{p+1} d_{v}\left(S_{p+1} \widetilde{z}\right) .
$$

Let $\left(y_{0}, \ldots, y_{p}\right) \in \mathfrak{g}^{p+1}$. By definition of $d_{h}$, we have

$$
\begin{aligned}
d_{h} z\left(y_{0}, \ldots, y_{p}\right)= & \sum_{i=0}^{p}(-1)^{i} y_{i} \cdot z(\underline{y}(i)) \\
& +\sum_{0 \leq r<s \leq p}(-1)^{r+s} z\left(\left[y_{r}, y_{s}\right], y_{1}, \ldots, \hat{y}_{r}, \ldots, \hat{y}_{s}, \ldots\right) .
\end{aligned}
$$

On the other hand, by Proposition 4.13

$$
(-1)^{p+1} d_{v}\left(S_{p+1} \widetilde{z}\right)\left(y_{0}, \ldots, y_{p}\right)=-\sum_{i=0}^{p}(-1)^{i} y_{i}^{*} \cdot z(\underline{y}(i)) .
$$

Now, $y_{i}-y_{i}^{*}=y_{i}^{+}$. The corollary follows by adding (4.16) and (4.17) and by comparing the result with the definition of $d_{+} z$. 


\section{The Spectral Sequence}

5.1. Spectral sequence generalities. In this section we recall some standard facts about spectral sequences. Our basic source is Chapter 5 of [Wei], but we have modified some of the definitions for convenience. See also Chapter XX.9 of [Lan.

Suppose $C=\bigoplus_{n \geq 0} C^{n}$ is a graded cochain complex with differential $d$. We also assume that $C$ has a decreasing filtration (compatible with $d$ ). That is, for each $n$, we have

$$
F_{0} C^{n} \supset F_{1} C^{n} \supset \cdots .
$$

The filtration is extended to negative indices by setting $F_{p} C^{n}=F_{0} C^{n}$ for $p<0$. We also assume that for each $n$ there exists some $r$ (depending on $n$ ) such that $F_{r} C^{n}=$ 0 . Let $B^{n} \subset Z^{n}$ denote the spaces of coboundaries and cocycles (respectively) in $C^{n}$. These spaces are filtered by setting $F_{p} B^{n}:=F_{p} C^{n} \cap B^{n}$ and $F_{p} Z^{n}:=$ $F_{p} C^{n} \cap Z^{n}$. The cohomology $H^{n}(C)$ is filtered by $F_{p} H^{n}(C)=\left(F_{p} Z^{n}+B^{n}\right) / B^{n}$.

Define

$$
E_{0}^{p q}=\operatorname{gr}^{p} C^{p+q}:=F_{p} C^{p+q} / F_{p+1} C^{p+q},
$$

and let $\pi_{p}: F_{p} C^{p+q} \rightarrow E_{0}^{p q}$ denote the projection.

For each $r, n, p \geq 0$ let

$$
F_{p} C^{n}(r)=\left\{c \in F_{p} C^{n} \mid d c \in F_{p+r} C^{n+1}\right\} .
$$

Thus, $F_{p} C^{n}=F_{p} C^{n}(0) \supset F_{p} C^{n}(1) \supset \cdots$. We define subspaces of $F_{p} C^{p+q}$ : first,

$$
Z_{r}^{p q}:=F_{p} C^{p+q}(r)+F_{p+1} C^{p+q} \supset B_{r}^{p q}:=d F_{p-r+1} C^{p+q-1}(r-1)+F_{p+1} C^{p+q} .
$$

Our assumption on the filtration means that for $r$ sufficiently large, $Z_{r}^{p q}=Z_{\infty}^{p q}$ and $B_{r}^{p q}=B_{\infty}^{p q}$, where by definition

$$
Z_{\infty}^{p q}:=F_{p} Z^{p+q}+F_{p+1} C^{p+q} \supset B_{\infty}^{p q}:=F_{p} B^{p+q}+F_{p+1} C^{p+q} .
$$

There are inclusions of these spaces:

$$
\begin{aligned}
0 & =B_{0}^{p q} \subset \cdots \subset B_{r}^{p q} \subset B_{r+1} \subset \cdots \subset B_{\infty}^{p q} \subset Z_{\infty}^{p q} \subset \cdots \subset Z_{r}^{p q} \subset Z_{r-1}^{p q} \subset \cdots \subset Z_{0}^{p q} \\
& =E_{0}^{p q} .
\end{aligned}
$$

The $r$-th page of the spectral sequence is defined to be

$$
E_{r}^{p q}=Z_{r}^{p q} / B_{r}^{p q}=\frac{F_{p} C^{p+q}(r)+F_{p+1} C^{p+q}}{d F_{p-r+1} C^{p+q-1}(r-1)+F_{p+1} C^{p+q}} .
$$

An equivalent definition of the $r$-th page is sometimes more convenient. Given subspaces $A, B, C$ of a vector space $V$, with $A \supset B$, we have a natural isomorphism

$$
\frac{A}{(A \cap C)+B} \rightarrow \frac{A+C}{B+C} \text {. }
$$

Applied to our situation, with $A=F_{p} C^{p+q}(r), B=d F_{p-r+1} C^{p+q-1}(r-1)$, and $C=F_{p+1} C^{p+q}$, we find that $A \cap C=F_{p+1} C^{p+q}(r-1)$, so we obtain a natural isomorphism

$$
E_{r}^{p q} \cong \frac{F_{p} C^{p+q}(r)}{G_{r}^{p q}}
$$

where $G_{r}^{p q}=F_{p+1} C^{p+q}(r-1)+d F_{p-r+1} C^{p+q-1}(r-1)$. Using this second description, we define a differential $d_{r}: E_{r}^{p q} \rightarrow E_{r}^{p+r, q-r+1}$ by

$$
d_{r}\left(c+G_{r}^{p q}\right)=d c+G_{r}^{p+r, q-r+1} .
$$


The following proposition describes one of the key properties of spectral sequences.

Proposition 5.4. Under the surjection $\pi_{p}: Z_{r}^{p q} \rightarrow E_{r}^{p q}$, the inverse image of $\operatorname{ker} d_{r}\left(\right.$ resp. $\left.\operatorname{im} d_{r}\right)$ is $Z_{r+1}^{p q}$ (resp. $\left.B_{r+1}^{p q}\right)$. Hence $\pi_{p}$ induces an isomorphism

$$
E_{r+1}^{p q}=Z_{r+1}^{p q} / B_{r+1}^{p q} \rightarrow H\left(E_{r}, d_{r}\right)^{p q} .
$$

We omit the proof.

Remark 5.5. For $r=0$ and $r=1$, the definitions (5.1) and (5.3) of the $r$-th page are identical. In particular, $G_{r}^{p q}=B_{r}^{p q}$, and $d_{r}\left(z+B_{r}^{p q}\right)=d z+B_{r}^{p q}$. However, the analogous assertion is false for $r>1$.

The spectral sequence is said to degenerate at $E_{r}$ if for all $p, q$, we have $Z_{r}^{p q}=Z_{\infty}^{p q}$ and $B_{r}^{p q}=B_{\infty}^{p q}$. This is equivalent to the vanishing of all differentials $d_{s}, s \geq r$, and implies that $E_{r}^{p q}=E_{\infty}^{p q}$.

Observe that

$$
\operatorname{gr}^{p} H^{p+q}(C) \cong \frac{F_{p} Z^{p+q}+B^{p+q}}{F_{p+1} Z^{p+q}+B^{p+q}} \cong \frac{F_{p} Z^{p+q}}{F_{p} B^{p+q}+F_{p+1} Z^{p+q}} .
$$

The composition

$$
F_{p} Z^{p+q} \rightarrow Z_{\infty}^{p q} \rightarrow E_{\infty}^{p q}
$$

induces an isomorphism $\operatorname{gr}^{p} H^{p+q}(C) \rightarrow E_{\infty}^{p q}$.

Suppose that $C$ has a graded algebra structure such that the differential $d$ satisfies $d\left(c c^{\prime}\right)=(d c) c^{\prime}+(-1)^{p} c\left(d c^{\prime}\right)$ for $c \in C^{p}, c^{\prime} \in C$. Then $H(C)$ is a graded algebra, and gr $H(C)$ is a bigraded algebra. Moreover, each $E_{r}$ has the structure of a bigraded algebra, and the map gr $H(C) \rightarrow E_{\infty}$ is an isomorphism of bigraded algebras.

5.2. Edge maps. Suppose that $F_{p+1} C^{p}=0$ for all $p$. Suppose also that $r \geq 2$; in this case $F_{p} C^{p}(r)=F_{p} Z^{p}$. Consider the map

$$
\epsilon_{p}: E_{r}^{p 0}=\frac{F_{p} Z^{p}}{d F_{p-r+1} C^{p-1}(r-1)} \rightarrow E_{\infty}^{p 0}=\frac{F_{p} Z^{p}}{F_{p} B^{p}}=\operatorname{gr}^{p} H^{p}(C) \subset H^{p}(C)
$$

The first map $E_{r}^{p 0} \rightarrow E_{\infty}^{p 0}$ is surjective. If the spectral sequence degenerates at $E_{r}$, we obtain

$$
E_{r}^{p 0}=\operatorname{gr}^{p} H^{p}(C) \hookrightarrow H^{p}(C) .
$$

Next, making use of the fact that if $r>0$, then $F_{1-r} C^{q}=C^{q}$, we have an edge map

$$
\epsilon_{q}: H^{q}(C)=\frac{Z^{q}}{B^{q}} \rightarrow \frac{Z^{q}}{B^{q}+F_{1} Z^{q}}=E_{\infty}^{0 q} \hookrightarrow E_{r}^{0 q}=\frac{F_{0} C^{q}(r)}{d F_{0} C^{q-1}(r-1)+F_{1} C^{q}(r-1)} .
$$

Here the first map is surjective. If the spectral sequence degenerates at $E_{r}$, we obtain a surjection

$$
H^{q}(C) \rightarrow \operatorname{gr}^{0} H^{q}(C)=E_{r}^{0 q}
$$


5.3. The product structure. Let $A^{p}=E_{\infty}^{p 0}, A=\bigoplus_{p} A^{p}, B^{q}=E_{\infty}^{0 q}$, and $B=$ $\bigoplus B^{q}$. Write $A^{+}=\bigoplus_{p>0} A^{p}$. We endow the tensor product $A \otimes B$ with an algebra structure such that

$$
\left(a_{1} \otimes b_{1}\right) \cdot\left(a_{2} \otimes b_{2}\right)=(-1)^{q_{1} p_{2}} a_{1} a_{2} \otimes b_{1} b_{2},
$$

for $b_{1} \in B^{q_{1}}$ and $a_{2} \in A^{p_{2}}$.

The edge maps give an inclusion $A \hookrightarrow H(C), A^{p}=\operatorname{gr}^{p} H^{p}(C) \subset H^{p}(C)$. Similarly, the edge maps give a surjection $H(C) \rightarrow B, H^{q}(C) \rightarrow \operatorname{gr}^{0} H^{q}(C)=B^{q}$. Let $J$ denote the kernel of the map $H(C) \rightarrow B$; then $J=\bigoplus_{q} F_{1} H^{q}(C)$.

Proposition 5.8. Suppose that the multiplication map $A \otimes B \rightarrow E_{\infty}$ is an algebra isomorphism. Then:

(1) The ideal $J$ is equal to the ideal of $H(C)$ generated by $A^{+}$.

(2) If $b_{1}, \ldots, b_{n}$ are homogeneous elements of $H(C)$ whose images under the map $H(C) \rightarrow B$ form a basis of $B$, then these elements form an $A$-module basis of $H(C)$. Hence if $\operatorname{dim} B=n$ is finite, then $H(C)$ is a free $A$-module of rank $n$.

Proof. (1) Let $J^{\prime}$ denote the ideal $A^{+} H(C)$. We want to show that $J^{\prime}=J$. Since $A^{+} \subset F_{1} H(C)$, we have $J^{\prime} \subset J$. For the reverse inclusion, it suffices to show that if $h \in F_{p} H^{q}(C)$ with $p \geq 1$, then $h \in J^{\prime}$. We use downward induction on $p$. If $p>q$, then $F_{p} H^{q}(C)=0$, so the result holds. Now suppose the result holds for elements of $F_{p+1} H^{q}(C)$. Let $\bar{h}$ denote the image of $h$ in $E_{\infty}^{p, q-p}$. By hypothesis we can write $\bar{h}=\sum a_{q} \beta_{q}$ for some elements $a_{q} \in A^{p}$ and $\beta_{q} \in B^{q-p}$. Choose elements $b_{q} \in H^{q-p}(C)$ mapping to $\beta_{q} \in B$. Then $h-\sum a_{q} b_{q} \in F_{p+1} H^{q}(C)$ is in $J^{\prime}$ by the inductive hypothesis. Since $\sum a_{q} b_{q} \in J^{\prime}$, we conclude $h \in J^{\prime}$, as desired.

(2) Let $b_{1}, \ldots, b_{n}$ be as in the statement of the proposition, let $d_{q}$ denote the degree of $b_{q}$, and let $\bar{b}_{q}$ denote its image in $B^{d_{q}} \cong F_{0} H^{d_{q}}(C) / F_{1} H^{d_{q}}(C)$. The argument that the $b_{q}$ generate $H(C)$ as an $A$-module is similar to the proof of (1), and we omit the details. To show that the $b_{q}$ are linearly independent over $A$, suppose we have a relation $\sum_{q} a_{q} b_{q}=0$ with not all $a_{q}=0$. We may assume that each term $a_{q} b_{q}$ has the same degree, which we denote $n$; then the degree of $a_{q}$ is $c_{q}:=n-d_{q}$. Let $c$ denote the smallest of the $c_{q}$ for which $a_{q} \neq 0$. Then each term $a_{q} b_{q}$ lies in $F_{c} H^{n}(C)$. By definition of the product on $E_{\infty}$, the image of $\sum_{q} a_{q} b_{q}$ in $E_{\infty}^{c, n-c} \cong F_{c} H^{n}(C) / F_{c+1} H^{n}(C)$ is $\sum_{c_{q}=c} a_{q} \bar{b}_{q}$. Since the multiplication map $A \otimes B \rightarrow E_{\infty}$ is an isomorphism, this implies $\sum_{c_{q}=c} a_{q} \otimes \bar{b}_{q}$ is zero in $A \otimes B$. As the $\bar{b}_{q}$ are linearly independent, this implies that all the $a_{q}$ with $c_{q}=c$ must be 0 , contradicting our choice of $c$. We conclude that the $b_{q}$ are linearly independent, as desired.

\section{The Relative Hochschild-Serre spectral Sequence}

The following theorem shows the existence of the Hochschild-Serre spectral sequence and identifies the edge maps.

Theorem 6.1. Let $\mathfrak{g}$ be a Lie algebra. Let $\mathfrak{k}$ be a subalgebra of $\mathfrak{g}$, reductive in $\mathfrak{g}$, and let $I$ be an ideal of $\mathfrak{g}$. Let $I_{\mathfrak{k}}=I \cap \mathfrak{k}$. Let $M$ be a $\mathfrak{g}$-module.

(1) There is a spectral sequence converging to $H^{p+q}(\mathfrak{g}, \mathfrak{k} ; M)$, and an isomorphism $\psi$ :

$$
E_{2}^{p q} \stackrel{\psi}{\rightarrow} H^{p}\left(\mathfrak{g} / I, \mathfrak{k} / I_{\mathfrak{k}} ; H^{q}\left(I, I_{\mathfrak{k}} ; M\right)\right)
$$


(2) The edge morphism $E_{2}^{p 0} \rightarrow H^{p}(\mathfrak{g}, \mathfrak{k} ; M)$ corresponds under the isomorphism $\psi$ to the composition

$$
H^{p}\left(\mathfrak{g} / I, \mathfrak{k} / I_{\mathfrak{k}} ; M^{I}\right) \rightarrow H^{p}\left(\mathfrak{g}, \mathfrak{k} ; M^{I}\right) \rightarrow H^{p}(\mathfrak{g}, \mathfrak{k} ; M),
$$

where the first map is the pullback induced by the projection $(\mathfrak{g}, \mathfrak{k}) \rightarrow\left(\mathfrak{g} / I, \mathfrak{k} / I_{\mathfrak{k}}\right)$, and the second map is induced by the $\mathfrak{g}$-module map $M^{I} \rightarrow M$.

(3) The edge morphism $H^{q}(\mathfrak{g}, \mathfrak{k} ; M) \rightarrow E_{2}^{0 q}$ corresponds under the isomorphism $\psi$ to the pullback

$$
i^{*}: H^{q}(\mathfrak{g}, \mathfrak{k} ; M) \rightarrow H^{q}\left(I, I_{\mathfrak{k}} ; M\right)^{\mathfrak{g} / I} .
$$

Part (1) of this theorem is proved in Section 6.2. Part (2) is Proposition 6.6, and part (3) is Proposition 6.7. In Section 6.4 we will show that the spectral sequence is compatible with pairings of representations.

6.1. Some notation. We follow the notational conventions of Definition 4.5. We have

$$
F_{p} C^{p+q} \stackrel{\pi_{p}}{\rightarrow} E_{0}^{p q} \stackrel{\bar{s}_{p}}{\rightarrow} C^{p}\left(C^{q}\right) .
$$

The composition is $s_{p}$, and by Lemma 3.7 it induces the isomorphism $\bar{s}_{p}$. Let $\bar{s}$ denote $\oplus_{p} \bar{s}_{p}$.

6.2. The differential $d_{0}$. We begin by calculating the differential $d_{0}$.

Proposition 6.2. Under the isomorphism $\bar{s}$, the differential $d_{0}: E_{0}^{p q} \rightarrow E_{0}^{p, q+1}$ corresponds to $(-1)^{p} d_{v}$.

Proof. Let $e \in E_{0}^{p q}$, and choose $\hat{e} \in F_{p} C^{p+q}$ satisfying $\pi_{p} \hat{e}=e$. By definition, $d_{0} e=\pi_{p} d e$, so $\bar{s}_{p} d_{0} e=s_{p} d \hat{e}$. As $s_{p}$ is the restriction of $S_{p}$, we apply Lemma 4.3 and find

$$
\bar{s}_{p} d_{0} e=s_{p} d \hat{e}=S_{p} d \hat{e}=d_{h}\left(S_{p-1} \hat{e}\right)+(-1)^{p} d_{v}\left(S_{p} \hat{e}\right) .
$$

As $\hat{e} \in F_{p} C^{p+q}$, we have $S_{p-1} \hat{e}=0$ by Lemma 3.7. Therefore,

$$
\bar{s}_{p} d_{0} e=(-1)^{p} d_{v}\left(S_{j} \hat{e}\right)=(-1)^{p} d_{v} s_{p} \hat{e}=(-1)^{p} d_{v} \bar{s}_{p} e,
$$

proving the proposition.

The following diagram summarizes some of the relationships between the groups we are considering. The vertical arrows are inclusions, and the horizontal maps $\alpha$ and $\beta$ (defined by this diagram) are the surjections from cocycles to cohomology.

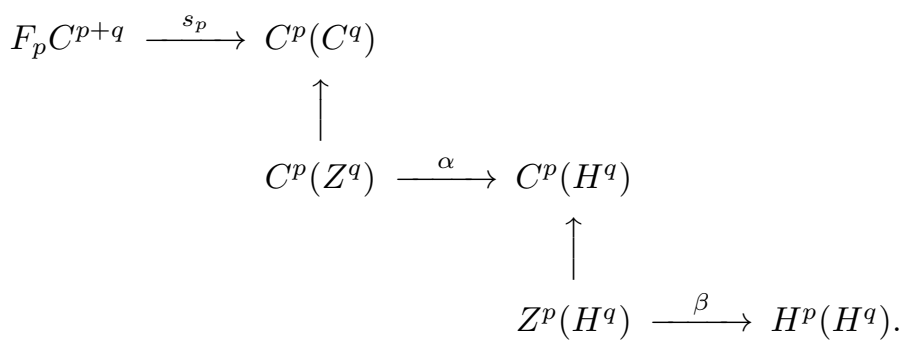

As a consequence of Proposition 6.2, we have:

Corollary 6.3. Under the surjective map $s_{p}$, the inverse image of $C^{p}\left(Z^{q}\right)$ (resp. $\left.C^{p}\left(B^{q}\right)\right)$ is $Z_{1}^{p q}$ (resp. $\left.B_{1}^{p q}\right)$. Hence the map $E_{1}^{p q} \rightarrow C^{p}\left(H^{q}\right)$ defined by $c+B_{1}^{p q} \mapsto$ $\alpha s_{p}(c)$ is an isomorphism. 
Proof. We have

$$
s_{p}^{-1}\left(C^{p}\left(Z^{q}\right)\right)=\pi_{p}^{-1}\left(\operatorname{ker} d_{0}\right)=Z_{1}^{p q},
$$

where the first equality is by Proposition 6.2 and the second by Proposition 5.4, A similar argument works to show $s_{p}^{-1}\left(C^{p}\left(B^{q}\right)\right)=B_{1}^{p q}$.

For $e=c+B_{1}^{p q} \in E_{1}^{p q}$, write $\phi(e)=\alpha s_{p}(c) \in C^{p}\left(H^{q}\right)$.

Proposition 6.4. Under the isomorphism $\phi: E_{1}^{p q} \rightarrow C^{p}\left(H^{q}\right)$, the spectral sequence differential $d_{1}$ corresponds to the Lie algebra cohomology differential $d_{+}$, i.e., $\phi\left(d_{1} e\right)=d_{+} \phi(e)$.

Proof. Let $e \in E_{1}^{p q}$, and let $\phi(e)=\alpha(z)$, with $z \in C^{p}\left(Z^{q}\right)$. Let $\widetilde{z} \in F_{p} C^{p+q}$ be the lift of $z$ defined in Definition 3.12 , Then $s_{p}(\widetilde{z})=z$, so by Corollary $6.3, \widetilde{z} \in Z_{1}^{p q}$. Observe that $e=\widetilde{z}+B_{1}^{p q}$; this follows by Corollary 6.3. since

$$
\phi\left(\widetilde{z}+B_{1}^{p q}\right)=\alpha\left(s_{p} \widetilde{z}\right)=\alpha(z)=\phi(e) .
$$

By Remark 5.5, $d_{1} e=d \widetilde{z}+B_{1}^{p+1, q}$, and therefore

$$
\phi\left(d_{1} e\right)=\phi\left(d \widetilde{z}+B_{1}^{p+1, q}\right)=\alpha s_{p+1}(d \widetilde{z})=\alpha\left(d_{+} z\right)=d_{+} \alpha(z)=d_{+} \phi(e),
$$

where the third equality is by Corollary 4.15, and the fourth is by definition of the map $d_{+}$on cohomology. This proves the result.

Theorem 6.5. If $c \in Z_{2}^{p q}$, then $\alpha s_{p}(c) \in Z^{p}\left(H^{q}\right)$. The map $Z_{2}^{p q} \rightarrow H^{p}\left(H^{q}\right)$ given by $c \mapsto \beta \alpha s_{p}(c)$ is surjective with kernel $B_{2}^{p q}$. Hence the induced map

$$
\psi: E_{2}^{p q} \rightarrow H^{p}\left(H^{q}\right)
$$

defined by

$$
\psi\left(c+B_{2}^{p q}\right)=\beta \alpha s_{p}(c)
$$

is an isomorphism.

Proof. We have

$$
Z_{1}^{p q} \stackrel{\pi_{p}}{\rightarrow} E_{1}^{p q} \stackrel{\phi}{\rightarrow} C^{p}\left(H^{q}\right),
$$

where $\pi_{p}$ is surjective and $\phi$ is an isomorphism. By Proposition 6.4 $\phi: \operatorname{ker}\left(d_{1}\right) \rightarrow$ $Z^{p}\left(H^{q}\right)$ and $\phi: \operatorname{im}\left(d_{1}\right) \rightarrow B^{p}\left(H^{q}\right)$ are isomorphisms. Thus, Proposition 5.4 implies that under $\phi \circ \pi_{p}$, the inverse image of $Z^{p}\left(H^{q}\right)$ is $Z_{2}^{p q}$ and the inverse image of $B^{p}\left(H^{q}\right)$ is $B_{2}^{p q}$. The result follows.

6.3. Edge maps. In this section we show that the edge maps are compatible with maps defined using the functorial properties of Lie algebra cohomology.

Since $H^{0}\left(I, I_{\mathfrak{k}} ; M\right)=M^{I}$, we have

$$
H^{p}\left(H^{0}\right)=H^{p}\left(\mathfrak{g} / I, \mathfrak{k} / I_{\mathfrak{k}} ; H^{0}\left(I, I_{\mathfrak{k}} ; M\right)\right)=H^{p}\left(\mathfrak{g} / I, \mathfrak{k} / I_{\mathfrak{k}} ; M^{I}\right) .
$$

There is a natural morphism

$$
\eta: H^{p}\left(H^{0}\right) \rightarrow H^{p}(\mathfrak{g}, \mathfrak{k} ; M)
$$

defined as the composition

$$
H^{p}\left(\mathfrak{g} / I, \mathfrak{k} / I_{\mathfrak{k}} ; M^{I}\right) \rightarrow H^{p}\left(\mathfrak{g}, \mathfrak{k} ; M^{I}\right) \rightarrow H^{p}(\mathfrak{g}, \mathfrak{k} ; M),
$$

where the first map is the pullback induced by the projection $(\mathfrak{g}, \mathfrak{k}) \rightarrow\left(\mathfrak{g} / I, \mathfrak{k} / I_{\mathfrak{k}}\right)$, and the second map is induced by the $\mathfrak{g}$-module map $M^{I} \rightarrow M$. More concretely, we can view $C\left(\mathfrak{g} / I, \mathfrak{k} / I_{\mathfrak{k}} ; M^{I}\right)$ as the subspace of elements $f \in C(\mathfrak{g}, \mathfrak{k} ; M)$ such that 
$f$ vanishes when any argument is in $I$, and such that the image of $f$ lies in $M^{I}$. We claim that

$$
F_{p} Z^{p}(\mathfrak{g}, \mathfrak{k} ; M)=Z^{p}\left(\mathfrak{g} / I, \mathfrak{k} / I_{\mathfrak{k}} ; M^{I}\right) .
$$

Since by definition of the filtration any element $g$ of the left hand side vanishes when any argument is in $I$, to verify the claim we only need to check that any such element has image in $M^{I}$. This follows because if $x_{0} \in I$, then

$$
0=d g\left(x_{0}, \ldots, x_{p}\right)=x_{0} g\left(x_{1}, \ldots, x_{p}\right) .
$$

Let $f \in Z^{p}\left(\mathfrak{g} / I, \mathfrak{k} / I_{\mathfrak{k}} ; M^{I}\right)$. The map $\eta$ takes the class of $f$ in $H^{p}\left(\mathfrak{g} / I, \mathfrak{k} / I_{\mathfrak{k}} ; M^{I}\right)$ to the class of the same element $f$, but now in $H^{p}(\mathfrak{g}, \mathfrak{k} ; M)$. We see that the image of $\eta$ lies in $F_{p} H^{p}(\mathfrak{g}, \mathfrak{k} ; M)$.

There is another morphism $H^{p}\left(H^{0}\right) \rightarrow H^{p}(\mathfrak{g}, \mathfrak{k} ; M)$ defined as the composition of $\psi^{-1}$ with the edge morphism $\epsilon_{p}$ :

$$
H^{p}\left(H^{0}\right) \stackrel{\psi^{-1}}{\rightarrow} E_{2}^{p 0} \stackrel{\epsilon_{p}}{\rightarrow} H^{p}(\mathfrak{g}, \mathfrak{k} ; M) .
$$

The next proposition shows that this morphism coincides with $\eta$.

Proposition 6.6. The following diagram commutes:

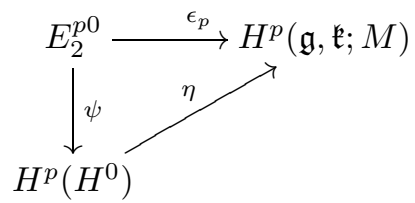

Proof. As observed in equation (5.6),

$$
E_{2}^{p 0}=\frac{F_{p} Z^{p}(\mathfrak{g}, \mathfrak{k} ; M)}{d F_{p-1} C^{p-1}(1)}
$$

Let $f \in F_{p} Z^{p}(\mathfrak{g}, \mathfrak{k} ; M)=Z^{p}\left(\mathfrak{g} / I, \mathfrak{k} / I_{\mathfrak{k}} ; M^{I}\right)$. Then $\epsilon_{p}$ takes the class of $f$ in $E_{2}^{p 0}$ to the class of $f$ in $H^{p}(\mathfrak{g}, \mathfrak{k} ; M)$. On the other hand, $s_{p} f$ is just $f$, now viewed as an element of $C^{p}\left(\mathfrak{g} / I, \mathfrak{k} / I_{\mathfrak{k}} ; C^{0}\left(I, I_{\mathfrak{k}} ; M\right)\right)$, and $\alpha s_{p} f$ is again $f$, but now viewed as an element of $Z^{p}\left(\mathfrak{g} / I, \mathfrak{k} / I_{\mathfrak{k}} ; M^{I}\right)$. Thus, $\psi$ takes the class of $f$ in $E_{2}^{p 0}$ to the class of $f$ in $H^{p}\left(\mathfrak{g} / I, \mathfrak{k} / I_{\mathfrak{k}} ; M^{I}\right)$. The discussion preceding the proposition shows that applying $\eta$ to this yields the class of $f$ in $H^{p}(\mathfrak{g}, \mathfrak{k} ; M)$. We conclude that the diagram commutes, as claimed.

We now consider the other edge morphism. By Proposition 2.11, the inclusion $i:\left(I, I_{\mathfrak{k}}\right) \rightarrow(\mathfrak{g}, \mathfrak{k})$ induces a pullback

$$
i^{*}: H^{q}(\mathfrak{g}, \mathfrak{k} ; M) \rightarrow H^{q}\left(I, I_{\mathfrak{k}} ; M\right)^{\mathfrak{g} / I}=H^{0}\left(\mathfrak{g} / I ; H^{q}\left(I, I_{\mathfrak{k}} ; M\right)\right)=H^{0}\left(H^{q}\right) .
$$

On the other hand, we have a morphism defined as the composition

$$
H^{q}(\mathfrak{g}, \mathfrak{k} ; M) \stackrel{\epsilon_{q}}{\rightarrow} E_{2}^{0 q} \stackrel{\psi}{\rightarrow} H^{0}\left(H^{q}\right) .
$$

The next proposition shows that these two morphisms agree.

Proposition 6.7. The following diagram commutes:

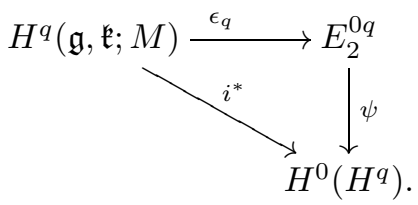


Proof. Let $c \in Z^{q}(\mathfrak{g}, \mathfrak{k} ; M)$. By definition (see (5.7)), the edge homomorphism $\epsilon_{q}$ takes the class of $c$ in $H^{q}(\mathfrak{g}, \mathfrak{k} ; M)$ to the class of $c$ in $E_{2}^{0 q}$, and $\psi$ takes this class to $\beta \alpha s_{0}(c)$. By Definition 3.2, $s_{0}(c) \in C^{0}\left(C^{q}\right)=C^{q}\left(I, I_{\mathfrak{k}} ; M\right)^{\mathfrak{k} / I_{\mathfrak{k}}}$ is the pullback $i^{*} c$. In addition, $i^{*} c \in Z^{q}\left(I, I_{\mathfrak{k}} ; M\right)^{\mathfrak{k} / I_{\mathfrak{k}}}$, and $\alpha s_{0}(c)$ is the cohomology class of $s_{0}(c)=$ $i^{*} c$ in $C^{0}\left(H^{q}\right)=H^{q}\left(I, I_{\mathfrak{k}} ; M\right)^{\mathfrak{k} / I_{\mathfrak{k}}}$. Further, $\alpha s_{0}(c)$ lies in $Z^{0}\left(H^{q}\right)=H^{0}\left(H^{q}\right)=$ $H^{q}\left(I, I_{\mathfrak{k}} ; M\right)^{\mathfrak{g} / I}$, and $\beta: Z^{0}\left(H^{q}\right) \rightarrow H^{0}\left(H^{q}\right)$ is the identity map. We conclude that the composition $\psi \circ \epsilon_{q}$ takes the cohomology class of $c$ to the cohomology class of $i^{*} c$, so the diagram commutes.

6.4. Pairings. A pairing of $\mathfrak{g}$-modules induces a pairing on spectral sequences. In this section we show that the basic result about these pairings ( $\mathrm{HS}]$, Theorem 5) extends to the relative situation.

Given a $\mathfrak{g}$-module $M$, write $E_{r}^{p q}(M)$ for the corresponding Hochschild-Serre spectral sequence. Write

$$
H^{p}\left(H^{q}(M)\right)=H^{p}\left(\mathfrak{g} / I, \mathfrak{k} / I_{\mathfrak{k}} ; H^{q}\left(I, I_{\mathfrak{k}} ; M\right)\right),
$$

and let $\psi_{M}: E_{2}^{p q}(M) \rightarrow H^{p}\left(H^{q}(M)\right)$ denote the isomorphism of Theorem 6.5.

Suppose $M, N$ and $P$ are $\mathfrak{g}$-modules with a $\mathfrak{g}$-module map $M \otimes N \rightarrow P$. There is a "cup product" pairing

$$
C^{p}(\mathfrak{g} ; M) \otimes C^{q}(\mathfrak{g} ; N) \rightarrow C^{p+q}(\mathfrak{g} ; P)
$$

taking $a \otimes b$ to $a \cup b$ (the formula is given in [HS], p. 592). As verified by Hochschild and Serre, this product is compatible with the differential $d$ and the action $\theta_{z}$ for $z \in \mathfrak{g}$, in that

$$
d(a \cup b)=d a \cup b+(-1)^{p} a \cup d b
$$

and

$$
\theta_{z}(a \cup b)=\theta_{z} a \cup b+a \cup \theta_{z} b .
$$

The cup product pairing induces a pairing on the spaces of relative cochains:

$$
C^{p}(\mathfrak{g}, \mathfrak{k} ; M) \otimes C^{q}(\mathfrak{g}, \mathfrak{k} ; N) \rightarrow C^{p+q}(\mathfrak{g}, \mathfrak{k} ; P) .
$$

Indeed, let $a \in C^{p}(\mathfrak{g}, \mathfrak{k} ; M)$ and $b \in C^{q}(\mathfrak{g}, \mathfrak{k} ; N)$. If $z \in \mathfrak{k}$, then $\theta_{z}(a \cup b)=\theta_{z} a \cup b+$ $a \cup \theta_{z} b=0$, and $i_{z}(a \cup b)=0$ (this is immediate from the cup product formula). The compatibility with the differential implies that the cup product pairing descends to cohomology, yielding a pairing

$$
H^{p}(\mathfrak{g}, \mathfrak{k} ; M) \otimes H^{q}(\mathfrak{g}, \mathfrak{k} ; N) \rightarrow H^{p+q}(\mathfrak{g}, \mathfrak{k} ; P) .
$$

Lemma 6.10. There is a pairing

$$
E_{r}^{p_{1}, q_{1}}(M) \otimes E_{r}^{p_{2}, q_{2}}(N) \rightarrow E_{r}^{p_{1}+p_{2}, q_{1}+q_{2}}(N),
$$

taking $[a] \otimes[b]$ to $[a \cup b]$ (the brackets denote the class of a cochain).

Proof. Write $A^{n}=C^{n}(\mathfrak{g}, \mathfrak{k} ; M), B^{n}=C^{n}(\mathfrak{g}, \mathfrak{k} ; N)$, and $D^{n}=C^{n}(\mathfrak{g}, \mathfrak{k} ; P)$. First, observe that the cup product satisfies

$$
F_{p_{1}} A^{p_{1}+q_{1}} \otimes F_{p_{2}} B^{p_{2}+q_{2}} \rightarrow F_{p_{1}+p_{2}} D^{p_{1}+p_{2}+q_{1}+q_{2}} .
$$

Next, the property $d(a \cup b)=d a \cup b+(-1)^{p} a \cup d b$ and (6.11) imply that

$$
F_{p_{1}} A^{p_{1}+q_{1}}(r) \otimes F_{p_{2}} B^{p_{2}+q_{2}}(r) \rightarrow F_{p_{1}+p_{2}} D^{p_{1}+p_{2}+q_{1}+q_{2}}(r) .
$$

The lemma follows in a straightforward way from equations (6.11) and (6.12). 
The pairing is compatible with the differential $d_{r}$ :

Lemma 6.13. Let $e \in E_{r}^{p_{1}+q_{1}}(M)$ and $e^{\prime} \in E_{r}^{p_{2}+q_{2}}(N)$. Then

$$
d_{r}\left(e \cup e^{\prime}\right)=\left(d_{r} e\right) \cup e^{\prime}+(-1)^{p_{1}+q_{1}} e \cup\left(d_{r} e^{\prime}\right) .
$$

Proof. The differential $d_{r}$ is calculated by choosing representative cochains and applying the differential $d$. The lemma is then a consequence of (6.9).

There is a pairing

$$
H^{q_{1}}\left(I, I_{\mathfrak{k}} ; M\right) \otimes H^{q_{2}}\left(I, I_{\mathfrak{k}} ; N\right) \rightarrow H^{q_{1}+q_{2}}\left(I, I_{\mathfrak{k}} ; P\right) .
$$

Since this is a $\mathfrak{g} / I$-module pairing, we obtain a corresponding pairing in $\left(\mathfrak{g} / I, \mathfrak{k} / I_{\mathfrak{k}}\right)$ cohomology (with notation as in (6.8)):

$$
H^{p_{1}}\left(H^{q_{1}}(M)\right) \otimes H^{p_{2}}\left(H^{q_{2}}(N)\right) \stackrel{\cup}{\rightarrow} H^{p_{1}+p_{2}}\left(H^{q_{1}+q_{2}}(P)\right) .
$$

Note that this pairing is derived from a pairing on the level of cochains.

The next result relates this pairing with the spectral sequence pairing, extending Theorem 5 of $[\mathrm{HS}]$ to the relative setting.

Proposition 6.15. Let $e \in E_{r}^{p_{1}+q_{1}}(M)$ and $e^{\prime} \in E_{r}^{p_{2}+q_{2}}(N)$. Then

$$
\psi_{P}\left(e \cup e^{\prime}\right)=(-1)^{p_{2} q_{1}} \psi_{M}(e) \cup \psi_{N}\left(e^{\prime}\right) .
$$

Proof. Keep the notation of the proof of Lemma 6.10. Let $a \in F_{p_{1}} A^{p_{1}+q_{1}}$ and $b \in F_{p_{2}} B^{p_{2}+q_{2}}$ represent $e$ and $e^{\prime}$, respectively. It suffices to prove that the pairing of cochains satisfies

$$
s_{p_{1}}(a) \cup s_{p_{2}}(b)=(-1)^{p_{2} q_{1}} s_{p_{1}+p_{2}}(a \cup b) .
$$

This follows from the calculation in the proof of Theorem 3 of [HS].

6.5. A tensor product decomposition. The natural pairing of the trivial representation $\mathbb{F}$ with itself induces a product on $E_{2}(\mathbb{F})$, making this space a bigraded ring. In this subsection, we describe a tensor product decomposition of $E_{2}(\mathbb{F})$, under a certain cohomological hypothesis. For simplicity, if the coefficient representation is the trivial representation $\mathbb{F}$, we will generally omit it from the notation. Thus, for example, we write $C^{n}(\mathfrak{g}, \mathfrak{k})$ and $H^{n}(\mathfrak{g}, \mathfrak{k})$ for $C^{n}(\mathfrak{g}, \mathfrak{k} ; \mathbb{F})$ and $H^{n}(\mathfrak{g}, \mathfrak{k} ; \mathbb{F})$, respectively, and $E_{2}$ for $E_{2}(\mathbb{F})$.

Unless otherwise stated, we will assume $H\left(\mathfrak{g} / I, \mathfrak{k} / I_{\mathfrak{k}}\right) \otimes H\left(I, I_{\mathfrak{k}}\right)$ is given the product structure defined by

$$
\left(a_{1} \otimes b_{1}\right)\left(a_{2} \otimes b_{2}\right)=(-1)^{p_{2} q_{1}}\left(a_{1} \cup a_{2}\right) \otimes\left(b_{1} \cup b_{2}\right),
$$

where $a_{2} \in H^{p_{2}}\left(\mathfrak{g} / I, \mathfrak{k} / I_{\mathfrak{k}}\right)$ and $b_{1} \in H^{q_{1}}\left(I, I_{\mathfrak{k}}\right)$. We will denote the product defined by the same formula except without the factor of $(-1)^{p_{2} q_{1}}$ by $*$, and refer to this as the "unsigned product".

Proposition 6.17. Suppose that for any $\mathfrak{g} / I$-module $V$, the inclusion $V^{\mathfrak{g} / I} \hookrightarrow V$ induces an isomorphism

$$
H\left(\mathfrak{g} / I, \mathfrak{k} / I_{\mathfrak{k}} ; V^{\mathfrak{g} / I}\right) \rightarrow H\left(\mathfrak{g} / I, \mathfrak{k} / I_{\mathfrak{k}} ; V\right) .
$$

Then there exists an algebra isomorphism

$$
\Psi: E_{2} \cong H\left(\mathfrak{g} / I, \mathfrak{k} / I_{\mathfrak{k}}\right) \otimes H\left(I, I_{\mathfrak{k}}\right)^{\mathfrak{g} / I}
$$


such that if $e_{1} \in E_{2}^{p 0}$ and $e_{2} \in E_{2}^{0 q}$, then

$$
\begin{aligned}
& \Psi\left(e_{1}\right)=\psi\left(e_{1}\right) \otimes 1, \\
& \Psi\left(e_{2}\right)=1 \otimes \psi\left(e_{2}\right) .
\end{aligned}
$$

Proof. First, there is a natural isomorphism

$$
f: C^{p}\left(\mathfrak{g} / I, \mathfrak{k} / I_{\mathfrak{k}}\right) \otimes H^{q}\left(I, I_{\mathfrak{k}}\right)^{\mathfrak{g} / I} \rightarrow C^{p}\left(\mathfrak{g} / I, \mathfrak{k} / I_{\mathfrak{k}} ; H^{q}\left(I, I_{\mathfrak{k}}\right)^{\mathfrak{g} / I}\right)
$$

defined by

$$
f(c \otimes \delta)\left(x_{1}, \ldots, x_{p}\right)=c\left(x_{1}, \ldots, x_{p}\right) \delta .
$$

This map induces a cohomology isomorphism (also denoted $f$ )

$$
f: H^{p}\left(\mathfrak{g} / I, \mathfrak{k} / I_{\mathfrak{k}}\right) \otimes H^{q}\left(I, I_{\mathfrak{k}}\right)^{\mathfrak{g} / I} \rightarrow H^{p}\left(\mathfrak{g} / I, \mathfrak{k} / I_{\mathfrak{k}} ; H^{q}\left(I, I_{\mathfrak{k}}\right)^{\mathfrak{g} / I}\right) .
$$

The space $H\left(\mathfrak{g} / I, \mathfrak{k} / I_{\mathfrak{k}} ; H\left(I, I_{\mathfrak{k}}\right)^{\mathfrak{g} / I}\right)$ has a ring structure. In fact, the definition of the product (which is made using the cohomology pairing induced by a pairing of representations) is such that if we equip $H\left(\mathfrak{g} / I, \mathfrak{k} / I_{\mathfrak{k}}\right) \otimes H\left(I, I_{\mathfrak{k}}\right)^{\mathfrak{g} / I}$ with the unsigned product, then $f$ is an algebra isomorphism. The isomorphism of (6.18) also respects the algebra structure, so the composition

$F: H\left(\mathfrak{g} / I, \mathfrak{k} / I_{\mathfrak{k}}\right) \otimes H\left(I, I_{\mathfrak{k}}\right)^{\mathfrak{g} / I} \stackrel{f}{\rightarrow} H\left(\mathfrak{g} / I, \mathfrak{k} / I_{\mathfrak{k}} ; H\left(I, I_{\mathfrak{k}}\right)^{\mathfrak{g} / I}\right) \rightarrow H\left(\mathfrak{g} / I, \mathfrak{k} / I_{\mathfrak{k}} ; H\left(I, I_{\mathfrak{k}}\right)\right)$ is an algebra isomorphism.

We now consider

$$
F: H^{p}\left(\mathfrak{g} / I, \mathfrak{k} / I_{\mathfrak{k}}\right) \otimes H^{q}\left(I, I_{\mathfrak{k}}\right)^{\mathfrak{g} / I} \rightarrow H^{p}\left(\mathfrak{g} / I, \mathfrak{k} / I_{\mathfrak{k}} ; H^{q}\left(I, I_{\mathfrak{k}}\right)\right)
$$

in case $p=0$ or $q=0$. If $p=0$, then $H^{0}\left(\mathfrak{g} / I, \mathfrak{k} / I_{\mathfrak{k}}\right)=\mathbb{F}$ and $H^{0}\left(\mathfrak{g} / I, \mathfrak{k} / I_{\mathfrak{k}} ; H^{q}\left(I, I_{\mathfrak{k}}\right)\right)$ $=H^{q}\left(I, I_{\mathfrak{k}}\right)^{\mathfrak{g} / I}$, and we have

$$
F(1 \otimes b)=b
$$

On the other hand, if $q=0$, then $H^{0}\left(I, I_{\mathfrak{k}}\right)^{\mathfrak{g} / I}=\mathbb{F}$ and $H^{p}\left(\mathfrak{g} / I, \mathfrak{k} / I_{\mathfrak{k}} ; H^{0}\left(I, I_{\mathfrak{k}}\right)\right)=$ $H^{p}\left(\mathfrak{g} / I, \mathfrak{k} / I_{\mathfrak{k}}\right)$, and we have

$$
F(a \otimes 1)=a .
$$

Let $\nu$ denote the inverse of $F$, and define $\Psi$ as the composition

$$
\Psi: E_{2} \stackrel{\psi}{\rightarrow} H\left(\mathfrak{g} / I, \mathfrak{k} / I_{\mathfrak{k}} ; H\left(I, I_{\mathfrak{k}}\right)\right) \stackrel{\nu}{\rightarrow} H\left(\mathfrak{g} / I, \mathfrak{k} / I_{\mathfrak{k}}\right) \otimes H\left(I, I_{\mathfrak{k}}\right)^{\mathfrak{g} / I} .
$$

The map $\Psi$ is a vector space isomorphism (since it is the composition of two such). Let $e_{1} \in E_{2}^{p_{1}, q_{1}}$ and $e_{2} \in E_{2}^{p_{2}, q_{2}}$. Then, applying Proposition 6.15] we see

$$
\begin{aligned}
\Psi\left(e_{1} e_{2}\right) & =\nu \psi\left(e_{1} e_{2}\right)=(-1)^{p_{2} q_{1}} \nu\left(\psi\left(e_{1}\right) \psi\left(e_{2}\right)\right) \\
& =(-1)^{p_{2} q_{1}} \nu\left(\psi\left(e_{1}\right)\right) * \nu\left(\psi\left(e_{2}\right)\right)=\Psi\left(e_{1}\right) \Psi\left(e_{2}\right) .
\end{aligned}
$$

Hence $\Psi$ is compatible with products, so it is an algebra isomorphism.

Finally, since $\Psi=\nu \psi$, equation (6.19) states that $\nu \psi\left(e_{1}\right)=\psi\left(e_{1}\right) \otimes 1$. Since $F$ and $\nu$ are inverses, this is equivalent to the equation $\psi\left(e_{1}\right)=F\left(\psi\left(e_{1}\right) \otimes 1\right)$, which follows from (6.22). The verification of (6.20) is similar.

Corollary 6.23. Keep the assumptions of Proposition 6.17 .

(1) There is an isomorphism

$$
H^{*}\left(\mathfrak{g} / I, \mathfrak{k} / I_{\mathfrak{k}}\right) \otimes H^{*}\left(I, I_{\mathfrak{k}}\right)^{\mathfrak{g} / I} \rightarrow E_{2}
$$

taking $a \otimes b$ to $\psi^{-1}(a) \psi^{-1}(b)$. 
(2) Suppose that the spectral sequence degenerates at $E_{2}$. Then the pullback map $\pi^{*}: H\left(\mathfrak{g} / I, \mathfrak{k} / I_{\mathfrak{k}}\right) \rightarrow H(\mathfrak{g}, \mathfrak{k})$ is injective and the pullback map $i^{*}: H(\mathfrak{g}, \mathfrak{k}) \rightarrow$ $H^{*}\left(I, I_{\mathfrak{k}}\right)^{\mathfrak{g} / I}$ is surjective. The kernel of $i^{*}$ is the ideal $J$ generated by elements of the form $\pi^{*}(a)$, where $a$ is a positive degree homogeneous element of $H\left(\mathfrak{g} / I, \mathfrak{k} / I_{\mathfrak{k}}\right)$.

Proof. Part (1) holds because the map (6.24) is the inverse of the map $\Psi$ from Proposition 6.17. For part (2), the injectivity of $\pi^{*}$ and surjectivity of $i^{*}$ follow because these maps are identified with edge maps (cf. Propositions 6.6 and 6.7) and the corresponding edge maps have this property. The statement about the kernel of $i^{*}$ then follows from Proposition 5.8 .

\section{The Belkale-Kumar Product}

In this section we apply the Hochschild-Serre spectral sequence to prove Theorem 1.1. which shows that the cohomology of a generalized flag variety, equipped with the Belkale-Kumar product, has a structure analogous to the cohomology of a fiber bundle.

Throughout this section $\mathfrak{g}$ is a complex semisimple Lie algebra. Let $G$ denote the corresponding adjoint group, with $B \supset H$ a Borel and maximal torus, respectively. Let $P$ be a parabolic subgroup of $G$ which contains $B$, and let $m=\operatorname{dim} H^{2}(G / P)$ (cohomology is taken with complex coefficients). For each $t=\left(t_{1}, \ldots, t_{m}\right) \in \mathbb{C}^{m}$, Belkale and Kumar construct a product $\odot_{t}$ on the space $H^{*}(G / P)$, and show that it is closely related to the geometric Horn problem. In [EG], it was shown that the ring $\left(H^{*}(G / P), \odot_{t}\right)$ is isomorphic to a certain relative Lie algebra cohomology ring.

To describe this ring we need some more notation. We denote the Lie algebra of an algebraic group by the corresponding fraktur letter. Let $\mathfrak{l}$ be the Levi subalgebra of $\mathfrak{p}$ containing $\mathfrak{h}$. Let $W$ and $W_{P}$ denote the Weyl groups of $\mathfrak{g}$ and $\mathfrak{l}$, respectively. Let $\left\{\alpha_{1}, \ldots, \alpha_{n}\right\} \subset \mathfrak{h}^{*}$ be the simple roots corresponding to the positive system for which the roots of $\mathfrak{b}$ are positive. Given $M \subset\{1, \ldots, n\}$, let $\mathfrak{l}_{M}$ be the Levi subalgebra generated by $\mathfrak{h}$ and the root spaces $\mathfrak{g}_{ \pm \alpha_{i}}$ for $i \in M$. Let $\mathfrak{u}_{M,+}$ (resp. $\mathfrak{u}_{M,-}$ ) be the subalgebra spanned by the positive (resp. negative) root spaces not contained in $\mathfrak{l}_{M}$. We assume that the simple roots are numbered so that $\mathfrak{l}=\mathfrak{l}_{I}$ for $I=\left\{\alpha_{m+1}, \ldots, \alpha_{n}\right\}$.

Let $t=\left(t_{1}, \ldots, t_{m}\right) \in \mathbb{C}^{m}$ and let $J(t)=\left\{1 \leq q \leq m: t_{q} \neq 0\right\}$. Let $K=I \cup J(t)$.

Given a subspace $V$ of $\mathfrak{g}$, let $V_{\Delta}$ denote the image of $V$ under the diagonal map $\mathfrak{g} \rightarrow \mathfrak{g} \times \mathfrak{g}$. Let

$$
\widetilde{\mathfrak{u}}_{K}=\left(\mathfrak{u}_{K,-} \times\{0\}\right)+\left(\{0\} \times \mathfrak{u}_{K,+}\right) .
$$

Define a subalgebra $\mathfrak{g}_{K}$ of $\mathfrak{g} \times \mathfrak{g}$ by

$$
\mathfrak{g}_{K}=\mathfrak{l}_{K, \Delta}+\widetilde{\mathfrak{u}}_{K} .
$$

Then $\widetilde{\mathfrak{u}}_{K}$ is an ideal of $\mathfrak{g}_{K}$, and $\mathfrak{g}_{K} / \widetilde{\mathfrak{u}}_{K} \cong \mathfrak{l}_{K}$. Moreover, $\mathfrak{g}_{K}$ contains $\mathfrak{l}_{\Delta}$ (since $\mathfrak{l}_{K}$ does).

For $t=0$, we denote $\widetilde{\mathfrak{u}}_{K}$ by $\widetilde{\mathfrak{u}}$, and note that $\widetilde{\mathfrak{u}}=\mathfrak{u}_{-} \times\{0\}+\{0\} \times \mathfrak{u}$, where $\mathfrak{u}$ is the nilradical of $\mathfrak{p}$, and $\mathfrak{u}_{-}$is the opposite nilradical. Thus, for $t=0, \mathfrak{g}_{K}=\mathfrak{l}_{\Delta}+\widetilde{\mathfrak{u}}$, and $H^{*}\left(\mathfrak{g}_{K}, \mathfrak{l}_{\Delta} ; \mathbb{C}\right) \cong H^{*}(\widetilde{\mathfrak{u}})^{L}$.

The following theorem describes the Belkale-Kumar product in terms of relative Lie algebra cohomology. The generic case and the case $t=0$ are established in BK]; the general case is in $\mathrm{EG}$.

Theorem 7.1. (1) The rings $\left(H^{*}(G / P), \odot_{t}\right)$ and $H^{*}\left(\mathfrak{g}_{K}, \mathfrak{l}_{\Delta} ; \mathbb{C}\right)$ are isomorphic. (2) $\left(H^{*}(G / P), \odot_{0}\right) \cong H^{*}(\widetilde{\mathfrak{u}})^{L}$. 
We now apply the Hochschild-Serre spectral sequence for relative Lie algebra cohomology. By Theorem 6.1, there is a spectral sequence converging to $H^{*}\left(\mathfrak{g}_{K}, \mathfrak{l}_{\Delta}\right)$ with $E_{2}$-term

$$
H^{*}\left(\mathfrak{g}_{K} / \widetilde{\mathfrak{u}}_{K}, \mathfrak{l}_{\Delta} ; H^{*}\left(\widetilde{\mathfrak{u}}_{K}, \mathbb{C}\right)\right) .
$$

Note that in this spectral sequence, the role of $I_{\mathfrak{k}}$ is played by $\widetilde{\mathfrak{u}}_{K} \cap \mathfrak{l}_{\Delta}=0$.

Proposition 7.3. The above spectral sequence degenerates at the $E_{2}$-term.

Proof. By standard facts concerning spectral sequences, it suffices to prove that the dimension of the $E_{2}$-term coincides with $\operatorname{dim}\left(H^{*}\left(\mathfrak{g}_{K}, \mathfrak{l}_{\Delta}\right)\right)$, which by Corollary 3.18 of [EG] is $\left|W^{P}\right|$. For this, we may identify $\mathfrak{g}_{K} / \widetilde{\mathfrak{u}}_{K}=\mathfrak{l}_{K, \Delta}$. We decompose

$$
H^{*}\left(\widetilde{\mathfrak{u}}_{K}, \mathbb{C}\right)=H^{*}\left(\widetilde{\mathfrak{u}}_{K}, \mathbb{C}\right)^{L_{K}} \oplus \sum_{i} V_{i},
$$

where the sum is over nontrivial irreducible representations of $L_{K}$ appearing in the space $H^{*}\left(\widetilde{\mathfrak{u}}_{K}, \mathbb{C}\right)$. For each $V_{i}$, there is a Hochschild-Serre spectral sequence converging to $H^{*}\left(\mathfrak{l}_{K}, \mathfrak{l} ; V_{i}\right)$ with $E_{2}$-term, $H^{*}\left(\mathfrak{l}_{K} / \mathfrak{z}_{K}, \mathfrak{l} / \mathfrak{z}_{K} ; H^{*}\left(\mathfrak{z}_{K}, V_{i}\right)\right)$. Since $\mathfrak{z}_{K}$ acts semisimply on $V_{i}, H^{*}\left(\mathfrak{z}_{K}, V_{i}\right)=0$ if $\mathfrak{z}_{K}$ acts nontrivially on $V_{i}$. Hence the modules $H^{*}\left(\mathfrak{z}_{K}, V_{i}\right)$ are a direct sum of nontrivial $\mathfrak{l}_{K} / \mathfrak{z}_{K}$-modules. Since $\mathfrak{l}_{K} / \mathfrak{z}_{K}$ is semisimple, Theorem 28.1 from [CE] implies that $H^{*}\left(\mathfrak{l}_{K} / \mathfrak{z}_{K}, \mathfrak{l} / \mathfrak{z}_{K} ; H^{*}\left(\mathfrak{z}_{K}, V_{i}\right)\right)=0$. Hence the $E_{2}$-term (17.2) coincides with

$$
H^{*}\left(\mathfrak{l}_{K}, \mathfrak{l}_{\Delta} ; H^{*}\left(\widetilde{\mathfrak{u}}_{K}, \mathbb{C}\right)^{L_{K}}\right) .
$$

This last space coincides with

$$
H^{*}\left(\mathfrak{l}_{K}, \mathfrak{l}\right) \otimes H^{*}\left(\widetilde{\mathfrak{u}}_{K}, \mathbb{C}\right)^{L_{K}} .
$$

It is well known that $\operatorname{dim} H^{*}\left(\mathfrak{l}_{K}, \mathfrak{l}\right)=\operatorname{dim} H^{*}\left(P_{K} / P\right)=\left|W_{P_{K}} / W_{P}\right|$, where $W_{P_{K}}$ is the Weyl group of $L_{K}$ (see [EG], Equation (3.10)). Also, by Theorem 5.14 from Kos1], $\operatorname{dim} H^{*}\left(\tilde{\mathfrak{u}}_{K}, \mathbb{C}\right)^{L_{K}}=\left|W / W_{P_{K}}\right|$. We conclude that the dimension of the $E_{2}$ term (7.2) is

$$
\left|W_{P_{K}} / W_{P}\right| \cdot\left|W / W_{P_{K}}\right|=\left|W^{P}\right|,
$$

and the proposition follows.

Theorem 7.5. (1) $H^{*}\left(\mathfrak{l}_{K}, \mathfrak{l}\right)$ is isomorphic to a subalgebra of $H^{*}\left(\mathfrak{g}_{K}, \mathfrak{l}_{\Delta}\right)$.

(2) Let $I_{+}=\sum_{q>0} H^{q}\left(\mathfrak{l}_{K}, \mathfrak{l}\right) H^{*}\left(\mathfrak{g}_{K}, \mathfrak{l}_{\Delta}\right)$, where we identify $H^{*}\left(\mathfrak{l}_{K}, \mathfrak{l}\right)$ with its image in $H^{*}\left(\mathfrak{g}_{K}, \mathfrak{l}_{\Delta}\right)$. Then

$$
H^{*}\left(\mathfrak{g}_{K}, \mathfrak{l}_{\Delta}\right) / I_{+} \cong H^{*}\left(\widetilde{\mathfrak{u}}_{K}, \mathbb{C}\right)^{L_{K}} .
$$

(3) The cohomology ring $H^{*}\left(P_{K} / P\right)$ with the usual cup product is isomorphic to a graded subalgebra $A$ of $\left(H^{*}(G / P), \odot_{t}\right)$. Further, the ring $\left(H^{*}\left(G / P_{K}\right), \odot_{0}\right) \cong$ $\left(H^{*}(G / P), \odot_{t}\right) / I_{+}$, where $I_{+}$is the ideal of $\left(H^{*}(G / P), \odot_{t}\right)$ generated by positive degree elements of $A$.

Proof. Let $p: \mathfrak{g}_{K} \rightarrow \mathfrak{g}_{K} / \widetilde{\mathfrak{u}}_{K} \cong \mathfrak{l}_{K}$ be the projection. By Propositions 6.6 and 7.3 and Corollary 6.23, $p^{*}$ is an injective ring homomorphism, which proves (1). Part (2) follows immediately from Corollary 6.23. Part (3) follows from parts (1) and (2) and Theorem 7.1 


\section{REFERENCES}

[BK] Prakash Belkale and Shrawan Kumar, Eigenvalue problem and a new product in cohomology of flag varieties, Invent. Math. 166 (2006), no. 1, 185-228. MR2242637(2007k:14097)

[BW] A. Borel and N. Wallach, Continuous cohomology, discrete subgroups, and representations of reductive groups, second ed., Mathematical Surveys and Monographs, vol. 67, American Mathematical Society, Providence, RI, 2000. MR1721403 (2000j:22015)

[CE] C. Chevalley and S. Eilenberg, Cohomology theory of Lie groups and Lie algebras, Trans. Amer. Math. Soc. 63 (1948), 85-124. MR0024908 (9:567a)

[EG] S. Evens and W. Graham, with an appendix jointly written with E. Richmond, The Belkale-Kumar cup product and relative Lie algebra cohomology, arXiv:1104.1415.

[HS] G. Hochschild and J.P. Serre, Cohomology of Lie algebras, Ann. of Math. (2) 57, (1953), 591-603. MR.0054581(14:943c)

[Kos1] Bertram Kostant, Lie algebra cohomology and the generalized Borel-Weil theorem, Ann. of Math. 74 (1961), 329-387. MR0142696 (26:265)

[Kos2] L Lie algebra cohomology and generalized Schubert cells, Ann. of Math. (2) 77 (1963), 72-144. MR0142697 (26:266)

[Lan] Serge Lang, Algebra, Revised third edition, Springer, 2002. MR1878556 (2003e:00003)

[Wei] Charles Weibel, An introduction to homological algebra, Cambridge University Press, Cambridge, 1994. MR.1269324 (95f:18001)

Department of Mathematics, University of Notre Dame, Notre Dame, Indiana 46556

E-mail address: sevens@nd.edu

Department of Mathematics, University of Georgia, Boyd Graduate Studies ReSearch Center, Athens, Georgia 30602

E-mail address: wag@math.uga.edu 